\title{
Probabilistic Lithium beam data analysis
}

\author{
R Fischer, E Wolfrum, J Schweinzer, ASDEX Upgrade Team \\ Max-Planck-Institut für Plasmaphysik, EURATOM Association, D-85748 Garching, \\ Germany \\ E-mail: rainer.fischer@ipp.mpg.de
}

\begin{abstract}
A probabilistic data analysis method was applied to analyze the spatially resolved beam emission intensities from the Lithium beam diagnostic at ASDEX Upgrade. The method allows to recover density profiles from the LiI emission profile with a spatial resolution of $5 \mathrm{~mm}$ and a temporal resolution of $50 \mu \mathrm{s}$. It is based on a probabilistic description of the measured data including absolutely calibrated measurement errors, a forward model for the simulation of the data given a density profile, and prior information about weak constraints on monotonicity. The density profile is parameterized by cubic spline polynomials. The method replaces the conventional approach applying an iterative (shooting) method to invert the density profile. The new probabilistic method allows to analyze low-density profiles since there is no need to fulfill an inner boundary condition or to pre-smooth measured data because no direct inversion occurs. Consistent profile uncertainty measures are provided for different purposes. Profiles in the edge region can be fully explored for any plasma regime and the profile pedestal is accessible up to $0.8 \times 10^{20} \mathrm{~m}^{-3}$. The advanced technique is demonstrated with low, medium and high density profiles at ASDEX Upgrade. The benefit of the achieved temporal resolution is shown with ELM resolved measurements. The dependence of the density profiles on electron temperature or impurity content is shown to be negligible.
\end{abstract}

PACS numbers: 52.70.-m, 52.25.Os, 07.05.Kf, 02.50.-r

Submitted to: Plasma Phys. Control. Fusion 


\section{Introduction}

Electron density profiles at the edge region of magnetically confined fusion plasmas are of major interest for understanding plasma-wall interaction physics as well as the transport barrier physics, e.g. in L- to H-mode transitions or edge localized modes. A routinely used method for measuring spatially and temporally resolved electron densities in the edge region of fusion plasmas (ASDEX Upgrade, W7-AS, Textor, CHS, and JET) is given by lithium beam impact excitation spectroscopy (Li-IXS) [1, 2, 3, 4, 5, 6, 7, 8].

The injected lithium atoms are excited and the neutral beam is attenuated by collisions with plasma particles. Therefore, the measured LiI $(2 \mathrm{~s}-2 \mathrm{p})$ resonance line at $670.8 \mathrm{~nm}$ depends on the plasma density. The spatial shape of the line emission profile is analyzed to reconstruct an electron density profile. The lithium beam attenuation and LiI emission line is modeled by solving balance equations of population and depopulation mechanisms by particle impact (electrons, hydrogen and impurity ions) and spontaneous emission [4].

The goal of the present work is to improve the analysis of the line emission profile by applying a probabilistic method to account for measurement and model uncertainties in a consistent way. The probabilistic method consists of two parts. The first part is given by the forward model calculating the occupation density of the $\operatorname{Li}(2 \mathrm{p})$ state and, therefore, the intensity of the line emission from a given density profile. This established physical model is successfully used since many years and provides the foundation for the present improvements. The second part is given by a probabilistic model for all measured and modeled quantities. The statistical model for the measured data provides a measure to distinguish data structures which are due to significant information from structure which is due to statistical fluctuations (noise). The probabilistic description of the modeled quantities is necessary for separating the significant information in the data from over-interpretation. Physical conditions and prior knowledge about parameters can be easily applied within a probabilistic approach. In contrast to the conventional method, the probabilistic method is conclusive in the sense that it is independent of an inner boundary condition or numerical stabilization techniques by data pre-smoothing. It provides density profiles for any plasma regime and is much more robust against numerical problems.

In addition to the analysis of diagnostic data in the most reliable way, the probabilistic method is useful for validation and combination of sets of diagnostics in a standardized way. The Integrated Data Analysis (IDA) approach provides a full probabilistic model including physical and statistical models of an integrated set of different diagnostics [9]. The goal of IDA is to combine data from heterogeneous and complementary diagnostics to consider all dependencies within and between diagnostics for obtaining validated and most reliable results. Though the lithium beam diagnostic is already combined within an IDA approach with the interferometry diagnostic at ASDEX Upgrade, the present work describes the progress obtained with the new probabilistic approach for the lithium beam only. Section 2 and 3 resume the forward 
model for the lithium emission data and the experimental set-up, respectively. Section 4 summarizes the conventional data analysis method and section 5 describes the probabilistic approach. The numerical costs are shown in section 6 . Results obtained with the conventional and the probabilistic approach are compared in section 7 . Section 8 shows results obtained with the temporal resolution of $50 \mu$ s. Section 9 shows the impact of the temperature profile and the impurity content on the density profile, and section 10 depicts a sensitivity study with respect to the regularization parameters. An exploration of profile uncertainty measures and their interpretative vulnerability is depicted in section 11. Section 12 summarizes.

\section{Lithium beam impact excitation spectroscopy}

Li-IXS is based on the observation of $\operatorname{LiI}$ radiation $(\operatorname{Li}(2 \mathrm{p}) \rightarrow \operatorname{Li}(2 \mathrm{~s}), \lambda=670.8 \mathrm{~nm})$ from neutral lithium atoms injected with an energy of $15-80 \mathrm{keV}$ into the plasma. Spatial line emission profiles are obtained from a collisional-radiative model including electron impact excitation, ionization and charge exchange processes. A system of coupled linear differential equations

$$
\begin{aligned}
& \frac{d N_{i}(z)}{d z}=\sum_{j=1}^{N_{\mathrm{Li}}}\left\{n_{\mathrm{e}}(z) a_{i j}\left(T_{\mathrm{e}}(z)\right)+b_{i j}\right\} N_{j}(z) \\
& N_{i}(z=0)=\delta_{1 i}
\end{aligned}
$$

describes the occupation densities of the excited states of Li. $z$ is the coordinate along the injected lithium beam where $z=0$ defines the entrance of the lithium beam into the plasma (first measured channel). $N_{i}$ is the occupation density of the $i$-th energy level, $N_{\mathrm{Li}}=9$ is the number of energy levels considered. The coefficient $a_{i j}(i \neq j)$ describes the population and de-population rate coefficient from level $i \rightarrow j$ due to collisions with electrons and protons of the plasma, respectively. Attenuation of the lithium state $i$ due to charge exchange and ionization in collisions with plasma particles and excitation to any other bound state are included in coefficient $a_{i i}$. The coefficients $b_{i j}$ are the Einstein coefficients of spontaneous emission. $n_{\mathrm{e}}$ and $T_{\mathrm{e}}$ are the electron density and temperature, respectively. At $z=0$ the lithium atoms are assumed to be in ground state where the boundary condition (2) has to be fulfilled. Details about the modeling of the lithium beam and the used atomic data bases can be found in $[4,10,11,12,13,14]$.

For any given density profile $n_{\mathrm{e}}(z)$ the initial value problem $(1,2)$ is solved with a variable-order, variable-step Adams method (NAG, 1984). The measured emission profile $d(z)$ of the $\operatorname{LiI}(2 \mathrm{~s}-2 \mathrm{p})$ transition is proportional to the occupation density $N_{2}(z)$ of the $\operatorname{Li}(2 \mathrm{p})$ state. The proportionality constant has to be determined.

\section{Experimental set-up}

Details of the experimental set-up can be found in $[15,5]$. The lithium injector for ASDEX Upgrade is capable of producing neutral lithium beams of 30-80 keV energy and 
2-4 mA equivalent current at the plasma boundary. The beam diameter is about $12 \mathrm{~mm}$ at this location. The beam enters the plasma radially near the equatorial midplane of the torus. The spatial resolution of $5 \mathrm{~mm}$ is determined by the aperture of the fiber optics used to transfer the emitted light to the combination of interference filters and photomultipliers for acquisition of the emission profile.

The temporal resolution is limited by the scanning frequency of $5 \mathrm{kHz}$ which was recently upgraded to $20 \mathrm{kHz}$. The increase of the temporal resolution by a factor of 4 was accompanied by a change of the set of interference filters from ones with $0.5 \mathrm{~nm}$ FWHM and $40 \%$ peak transmission to a set with $2 \mathrm{~nm}$ FWHM and $80 \%$ peak transmission. As the narrow filters had to be tilted for optimal performance at different beam velocities, this change of filter set resulted in an increase of the signal-to-noise ratio in most spatial channels by about one order of magnitude. The effective temporal resolution for the conventional data analysis tool is determined by the required signal-to-noise ratio of the emitted light. A temporal resolution of $5-20 \mathrm{~ms}$ is usually chosen by averaging over subsequent time frames.

The probabilistic approach does not need a predetermined signal-to-noise ratio to allow for a successful analysis. In case of a small signal-to-noise ratio the estimated density just shows a large estimation uncertainty.

The achieved temporal $(50 \mu \mathrm{s})$ and spatial $(5 \mathrm{~mm})$ resolution is comparable to the resolutions obtained with reflectometry measurements $(35 \mu \mathrm{s}$ and $\leq 10 \mathrm{~mm})[16]$ but providing density profiles from reflectometry measurements routinely poses a challenging task. Section 8 shows density profiles with a temporal resolution up to the technical limit of $50 \mu$ s obtained with the new probabilistic method.

To discriminate the signal from the background, the beam is chopped with a cycle time of $80 \mathrm{~ms}$ (56 ms beam on, $24 \mathrm{~ms}$ beam off). The background signal is averaged within the beam-off phase and linearly interpolated in-between.

\section{Conventional data analysis method}

The established method to evaluate electron density profiles from line emission profiles from the lithium beam is provided by the "IPP technique" [4]. This technique is inherently capable of deriving absolute $n_{\mathrm{e}}(z)$ profiles from the relative line emission profile without need for absolute calibration. The evaluation of density profiles employing this conventional technique is routinely used in ASDEX Upgrade. For selfconsistent density estimation it is crucial that the whole emission profile is observed $[7]$.

The conventional method is based on an algebraic rearrangement of the second differential equation obtaining an explicit equation for the density $n_{\mathrm{e}}$ as a function of the beam coordinate $z$ and of all occupation densities [4, 7]. Using this explicit equation the density profile is obtained by a stepwise integration of (1) starting at $z=0$. The absolute calibration constant $\alpha$ relating $d(z)$ to $N_{2}(z)$ is determined by "shooting" (iteration) for a solution for different values of $\alpha$ until a self-consistent 
solution is obtained. This can be achieved by either assuming a completely ionized beam at the last spatial channel or by enforcing that both, numerator and denominator in the explicit equation reach zero at the same radial position (singularity condition). This approach has been used for ASDEX Upgrade as a standard method and produces satisfactory results for most of the plasma scenarios. At JET the absolute calibration constant $\alpha$ is obtained routinely by fitting an inner boundary condition which may be adjusted with respect to an independent measurement of the electron density [7].

The major drawback of the conventional method is that the method works only if the singularity condition or an inner boundary condition of vanishing lithium beam intensity for the innermost spatial channel (equation (6) in [4]) can be fulfilled (or an independent density measurement exists). For small density regimes neither the beam is fully ionized at the innermost spatial channel nor the singularity appears. Therefore, for low-density plasma regimes density profiles could not be obtained. Additionally, the conventional method suffers from numerical problems close to the singularity as well as from statistical fluctuations (noise) in the data. The singularity condition is very sensitive on minor changes in $\alpha$. To extend the achievable spatial region for density evaluation and to improve numerical stability, the line emission data were spatially smoothed and temporally binned prior to the analysis in order to reduce the statistical noise in the data. However, spatial smoothing might result in loss of significant information provided by the data, e.g., at the emission maximum. Temporal binning reduces time resolution and is useful only for plasma conditions stationary in the time interval studied. Furthermore, the information about data errors are lost after applying pre-smoothing and, therefore, profile uncertainties reflecting data noise are no longer accessible.

\section{Bayesian probability theory}

To avoid the intrinsic problems of the conventional method and to tackle the measurement errors in a consistent way, a new probabilistic data analysis tool for the lithium beam diagnostic was developed. It is based on a probabilistic description of the measured data and a forward model for the simulation of the data from a given density profile. Within the framework of probability theory, the measured data are compared with the forward model describing the line emission for a given density profile. Since only forward modeling is involved no direct inversion of the noisy data is necessary. An example of the probabilistic technique applied to a Thomson scattering diagnostic can be found in [9] and references therein.

Special attention is given to the description of the measurement errors of the line emission signal, the error of the background measurement of the chopped lithium beam, and the uncertainty of the relative calibration of the spatial channels. An elaborate error assessment is crucial for recovering only the significant information in the measured data and for avoiding noise fitting. As a result, the uncertainty of the estimated density profile reflects all error sources encountered. 
In comparison to the conventional analysis, the new probabilistic analysis method has several advantages: For a self-consistent density estimation, it is not crucial to measure the whole emission profile. The method works for incomplete emission profiles and, therefore, for all density regimes. The proportionality parameter $\alpha$ is a fitting (nuisance) parameter which can be estimated jointly with the density profile. The iteration of the calibration constant $\alpha$ ("shooting") for solving the equations is obsolete. The existence of the singularity condition or an inner boundary condition by a completely ionized beam or an independent measurement is not necessary. Since there are no singularities in the forward calculation, there are no numerical instabilities due to small de-numerators or experimental noise. Therefore, there is no need for pre-smoothing or temporal binning of the data. This implies that consistent profile uncertainty measures can be provided.

Inferring an electron density profile $n_{\mathrm{e}}$ from measured data $d$ is an inverse problem which cannot be solved uniquely if the data are deteriorated by noise $\sigma$ or the information needed for modeling the data is incomplete. For the lithium beam diagnostic the data set consists of noisy signals from 35 spatially resolved light detectors and calibration data. Estimates and errors of $n_{\mathrm{e}}$ profiles have to comprise uncertainties encountered in both, the measured data and the model parameters which enter data interpretation. Probability theory provides a consistent framework for combining noisy data and incomplete or uncertain additional information relevant for the measurement. Probability theory as a logical inference concept, usually termed as Bayesian probability theory (BPT), is extensively used in various fields of plasma physics such as parameter estimation [17], reconstruction of electron energy distribution functions [18], separation of the signal from the background [19, 20], robust estimation and outlier treatment [21], model comparison and group analysis [22, 23], data validation [24] and experimental design [25]. The concept of Integrated Data Analysis (IDA) [9, 26] in the framework of BPT offers a unified way of combining all available information for single diagnostic data analysis as well as for the coherent combination of heterogeneous diagnostics. The present work is part of a larger effort of combining complementary diagnostics of ASDEX Upgrade in the IDA framework. A comprehensive probabilistic description of the individual diagnostics involved are an essential prerequisite for IDA. A recommended tutorial on BPT is given by Sivia [27].

The starting point of BPT is given by Bayes' theorem, which reads for the present application as:

$$
P\left(n_{\mathrm{e}} \mid d, \sigma, I\right)=\frac{P\left(d \mid n_{\mathrm{e}}, \sigma, I\right) P\left(n_{\mathrm{e}} \mid I\right)}{P(d \mid I)}
$$

Bayes' theorem is a consequence of the sum and product rules of probability theory. Eq. 3 relates the posterior probability distribution function (PDF) $P\left(n_{\mathrm{e}} \mid d, \sigma, I\right)$ to known quantities, namely, the likelihood $\operatorname{PDF} P\left(d \mid n_{\mathrm{e}}, \sigma, I\right)$ and the prior $\operatorname{PDF} P\left(n_{\mathrm{e}} \mid I\right) . P(d \mid I)$ is the evidence of the data which constitutes the normalization and will not affect the conclusions within the context of a given model. The evidence is an important quantity for model comparison because it evaluates how consistent the measured data are with 
the statistical and physical models provided.

The posterior PDF is the quantity to be inferred. It encodes all information to decide how reasonable a solution $n_{\mathrm{e}}$ is if the data $d$, the uncertainty of the data $\sigma$ and further information $I$ are given. The information $I$ abbreviates additional knowledge about the problem such as the physical model or constraints on $n_{\mathrm{e}}$. $n_{\mathrm{e}}$ is restricted by positivity constraints and can be further confined by monotonicity assumptions or correlations with other quantities such as $p_{e}=n_{e} * T_{e}$. For practical reasons, the posterior PDF is frequently characterized by two values of interest, namely the best estimate, often given by the maximum or mean value of the posterior PDF, and its reliability, given by the width. If the posterior PDF is unimodal, e.g. a Gaussian distribution, the estimate can be assigned to the most probable value of the PDF and a measure of the error is given by the standard deviation. For the case of non-Gaussian distributions the characterization of the posterior by only two values fails to summarize the results. An example is given by the combination of results from different, inconsistent measurements resulting in estimation uncertainties much smaller than the difference of the estimates. The result may be a bi- or multi-modal distribution. Such problems are often encountered in comparing results from different diagnostics and, indeed, initiated the present work of a thorough understanding of the lithium beam diagnostic (in combination with the interferometry diagnostic).

For applying Bayes' theorem the likelihood and the prior pdfs have to be quantified. The likelihood provides a measure to distinguish data structures which are due to significant information from structures which are due to statistical fluctuations or systematic uncertainties. The prior is necessary for reducing the space of possible solutions allowed by the likelihood to the significant information in the data. Priors should prevent physically unreasonable solutions or over-fitting.

\subsection{Likelihood}

The likelihood function describes the error statistics of the experiment. If the noise $\epsilon=d-D\left(n_{\mathrm{e}}\right)$ of a single measurement $d$ is assumed to be independent and normally distributed with zero mean $(\langle\epsilon\rangle=0)$ and variance $\left\langle\epsilon^{2}\right\rangle=\sigma^{2}$, the likelihood function is given by a Gaussian pdf. The Li-beam intensity data set used for estimating the density profile consists of $N_{s}=35$ spatial channels and $N_{t}$ consecutive time frames recorded every $50 \mu \mathrm{s}$ (200 $\mu \mathrm{s}$ for older shots). The combined likelihood of $N_{d}=N_{s} \cdot N_{t}$ data values with independent errors is given by the product of Gaussian likelihood pdfs

$$
\begin{aligned}
P\left(d \mid n_{\mathrm{e}}, \sigma, I\right) & =\frac{1}{\prod_{i j}^{N_{s}, N_{t}} \sqrt{2 \pi \sigma_{i j}^{2}}} \exp \left(-\frac{1}{2} \chi^{2}\right), \\
\chi^{2} & =\sum_{i j}^{N_{d}}\left(\frac{d_{i j}-D_{i}\left(n_{\mathrm{e}}\right)}{\sigma_{i j}}\right)^{2}
\end{aligned}
$$

in terms of the familiar $\chi^{2}$ misfit. $\sigma_{i j}$ is the standard deviation of the error of measurement $d_{i j}$ at spatial channel $i=1 . . N_{s}$ and at time $t_{j}, j=1 . . N_{t} . \quad D_{i}\left(n_{\mathrm{e}}\right)$ is 
the modeled line emission intensity at spatial channel $i$. It is assumed that the emission profiles of all consecutive time frames selected can be modeled with a unique density profile. If the density profile changes on a faster time scale, the number of time slices have to be reduced. For rapid density changes during an ELM onset, each time frame has to be analyzed separately. If the assumption of a stationary density profile over the combined time frames is not fulfilled, the residuals of the fitted data increase. Systematic deviances of the residuals for different time frames provide an easy to implement indicator for the necessity of a larger temporal resolution. The advantage of the present likelihood over temporal data binning by adding up the data within the time window is twofold. First, the uncertainty of the single datum is considered correctly. Second, systematic changes between different time points can be easily detected in the residuals.

To calculate the likelihood pdf for a given set of emission profiles we have to provide a model for the line emission and the uncertainties of the data.

\subsection{Lithium beam measurement}

The modeled lithium beam intensity $D$ due to emitted line radiation detected by the spatial channels is given by:

$$
D_{i}\left(n_{\mathrm{e}}\right)=\alpha s_{i} N_{2}\left(n_{\mathrm{e}}\left(z_{i}\right)\right) .
$$

The proportionality factor $\alpha$ accounts for imaging effects, the lithium beam intensity and the overall sensitivity of the detection system. Since the lithium beam intensity varies on a fast time scale due to space charge effects and changes in the neutral gas density between the beam generating box and the plasma entrance $(\approx$ meters $), \alpha$ cannot be determined independently but has to be estimated for each time frame from the measured line-emission profiles, even if an absolute calibration of the beam intensity would be possible. Therefore, the parameter $\alpha$ is estimated together with the parameters of the density profile $n_{\mathrm{e}}$. The parameters $s_{i}$ account for the relative sensitivities of the spatial channels for which calibration measurements are needed. The calibration measurement, described in section 5.4, is performed with the same experimental set-up as the lithium beam measurement in the plasma.

The signal $d_{i j}$ measured by an individual spatial channel $i$ and time point $j$ is given by the sum of the line emission intensity $D$, background intensity $D_{\mathrm{b}}$ and noise $\epsilon$, respectively:

$$
d_{i j}=D_{i}+D_{i, \mathrm{~b}}+\epsilon_{i j}
$$

In order to determine the background, the lithium beam is chopped:

$$
d_{i j, \mathrm{~b}}=D_{i, \mathrm{~b}}+\epsilon_{i j, \mathrm{~b}}
$$

For the present case of a measured background, the uncertainty of the estimated background has to be considered. This results in a modification of the $\chi^{2}$-term in the likelihood:

$$
\chi^{2}=\sum_{i j}^{N_{d}}\left[\frac{\left(d_{i j}-d_{i j, \mathrm{~b}}-D_{i}\left(n_{\mathrm{e}}\right)\right)^{2}}{\sigma_{i j}^{2}+\sigma_{i j, \mathrm{~b}}^{2}}\right]
$$


where $\sigma_{i j, \mathrm{~b}}$ is the uncertainty of the background estimate.

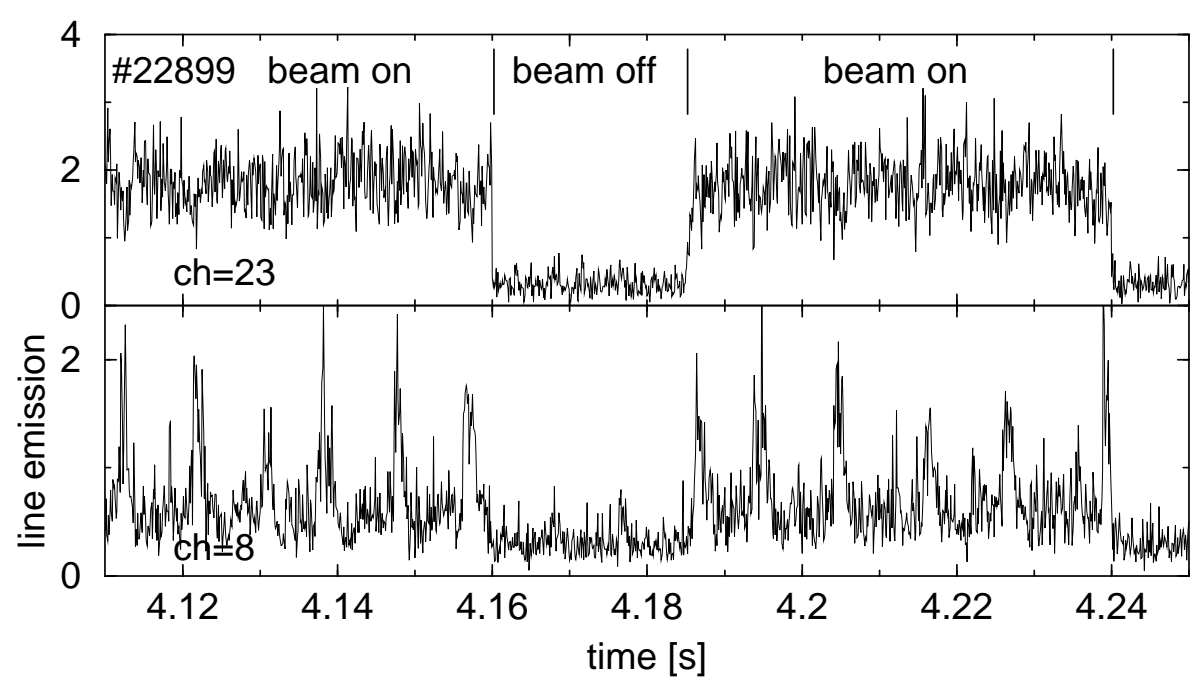

Figure 1. Time traces of the measured line emission intensity for channel numbers 8 and 23 for shot number \#22899.

Figure 1 shows time traces of the measured line emission intensity of channel numbers 8 and 23 for shot number \#22899. The time window comprises 2 beam-on and 1 beam-off (chopped) time intervals within an H-mode phase with type-I ELMs. The ELMs can be clearly seen as bursts of line emission. Channel 8 measures in the SOL and channel 23 measures close to the pedestal top where an ELM induced decrease of the line intensity is observed. Although ELMs occurred also within the beam-off phase, the background trace does not show an significant increase of the signal during the ELM. It follows that the background signal is not spoiled by the ELM. The background can be clearly estimated from the mean of the beam-off signal and the background uncertainty is given by the standard mean uncertainty.

The background consists mainly of bremsstrahlung radiation. Line radiation from tungsten was not observed because the power deposition at the first-wall area covered by the line-of-sight is negligible. Nevertheless, the recently installed broad-band interference filters cover part of a helium line. Figure 2 shows time traces of the measured line emission intensity of channel numbers 8 and 23 for shot number \#22845. This shot is comparable to \#22899 but the helium concentration is much larger. This shot was recorded shortly after a helium glow discharge for wall conditioning. Averaging the background signal is not appropriate for plasmas with non-stationary plasma conditions and large helium concentrations. For ELM-free regions the background is flat which allows to average the background signal. More sophisticated background estimation methods have to be developed for non-stationary plasma phases (ELMs) with large helium concentrations. The signal measured with the previous small-band filters did suffer much less from helium line emission. Fortunately, the helium concentration decreases rapidly after a couple of shots which allows to use the standard background 


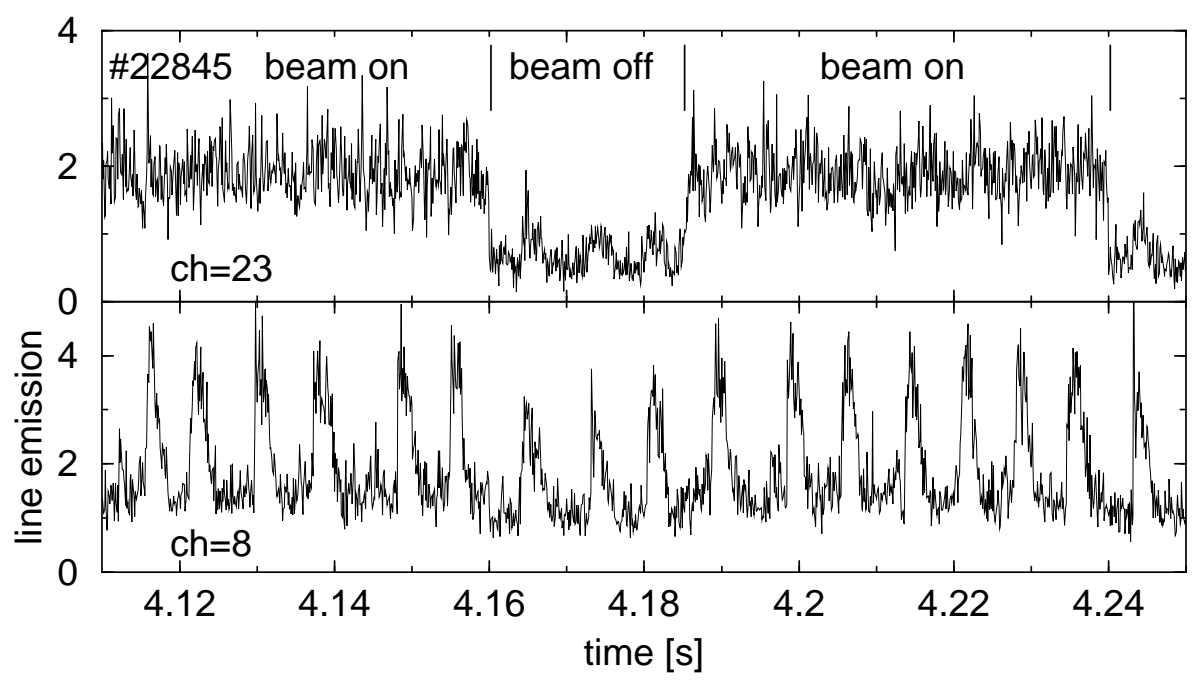

Figure 2. Time traces of the measured line emission intensity for channel numbers 8 and 23 for shot number \#22845 with large helium concentrations.

estimation approach routinely.

Non-stationary plasma conditions on time scales below the integration time of 200 or $50 \mu \mathrm{s}$, respectively, are not relevant. Therefore, small-scale plasma fluctuations are not resolved because they occur on much smaller time scales. Bremsstrahlung from such fluctuations do not have to be considered for the estimation of the background. Transient (blobby) effects on time scales larger than $50 \mu$ s will be subject of further studies.

\subsection{Error assessment}

A basic requirement for probabilistic data analysis is a comprehensive error assessment of the measured data and all nuisance parameters entering the forward model (data descriptive model). The measurement errors of the lithium emission intensities is derived from the calibration measurement with post-filling of the ASDEX Upgrade vessel with $\mathrm{H}_{2} / \mathrm{D}_{2}$ gas. Assuming Poisson statistics of a counting experiment the likelihood of measuring $x$ counts is given by

$$
p(x \mid \lambda)=\frac{\lambda^{x}}{x !} \exp (-\lambda)
$$

with mean intensity $\lambda$ which is identical to the variance of the Poisson distribution. If the true measurement $d$ suffers from an unknown offset $x_{0}$ and an unknown amplification factor $\xi$,

$$
d=\xi\left(x-x_{0}\right),
$$

the resulting likelihood again is a Poisson distribution with mean and variance shifted and scaled

$$
\langle d\rangle \quad=\xi\left(\lambda-x_{0}\right)
$$




$$
\left\langle d^{2}\right\rangle-\langle d\rangle^{2}=\xi^{2} \lambda
$$

From the calibration measurement with constant gas pressure the unknown offset and scale values can be derived for each of the 35 spatial lithium beam channels individually. The right panel of figure 3 summarizes the measured intensities in two histograms for beam on and beam off, respectively. The mean $m$ and variance $v$ values of the background (index 1) and signal (index 2) measurements provide four numbers which allow to calculate the four unknown quantities $x_{0}, \xi, \lambda_{1}$ and $\lambda_{2}$ from the four equations

$$
m_{1,2}=\xi\left(\lambda_{1,2}-x_{0}\right) \quad, \quad v_{1,2}=\xi^{2} \lambda_{1,2}
$$

namely

$$
\begin{aligned}
\xi & =\frac{v_{1}-v_{2}}{m_{1}-m_{2}} \\
\lambda_{1,2} & =v_{1,2} / \xi^{2} \\
x_{0} & =\lambda_{1}-m_{1} / \xi
\end{aligned}
$$

The solid lines depict the Poisson distribution (normalized to number of measurements) corresponding to the offset and mean values derived with the set of equations (15). The factorial in the Poisson distribution (10) is replaced by a Gamma-function, $x ! \rightarrow \Gamma(x+1)$, to allow for continuous $x$-values. For the measurement of the channel 18 shown in figure 3 the estimates for the offset is $x_{0}=3.0$ and for the scale $\xi=0.091$, obtaining a measured offset of $-\xi * x_{0}=-0.28$. The estimated mean of $\lambda_{1}=3.3$ and $\lambda_{2}=8.4$ show that one measures in the mean for each time frame only a few photons. The reason for the histogram not showing distinguished count numbers is the statistical behavior of the amplifiers in the measurement system. The Poisson distributions estimated for all 35 lithium channels describe the data reasonably well even for the asymmetric lowintensity cases with values of $\lambda_{1}=0.2-4$ and $\lambda_{2}=1-15$. Due to the small number of photons collected within one time frame the estimate of the measurement offset (section 5.2) and absolute calibration(section 5.4) are based on about 5000 time frames within $1 \mathrm{~s}$ to allow for reasonable statistics.

With the offset and scale values determined individually for the spatial channels, the number (actually not an integer but a real value due to amplifier noise) of measured photons can be estimated,

$$
n=\frac{d}{\tilde{\xi}}+x_{0},
$$

where $n$ is the number of measured photons and $d$ is the measured intensity. $\tilde{\xi}=\xi / 10$ because the signal amplification for the calibration measurement is chosen to be a factor of 10 larger than for the plasma measurement. If $n$ is sufficiently large $(n>10)$ the Poisson distribution is approximated reasonable well by a normal distribution with standard deviation $\sqrt{n}$. Therefore, the statistical error on the measured line intensity $d \pm \sigma$ is given by

$$
\sigma=\tilde{\xi} \sqrt{n}=\tilde{\xi} \sqrt{\frac{d}{\tilde{\xi}}+x_{0}}
$$


An independent check of the error statistics derived from the calibration measurement is given by the residuals between the measured intensities of the line emission in the plasma and the modeled intensities weighted with the statistical error (17). Indeed, figure 5 shows that the assumption of counting photons (Poisson statistics) and the validity of the estimated offset $x_{0}$ and scale $\xi$ is confirmed because the residuals of the fitted line emission profiles scatter independently in the order of magnitude of one.

The statistical error given in equation 17 is valid for the complete range of measured intensities. A small amount of the 35 spatial channels have a poor signal-to-noise ratio due to degradation of optical components. For the conventional analysis tool these degenerate channels have to be removed from the set of data to avoid systematic errors in the profiles. This data censor is not necessary for the new probabilistic technique since each datum is taken into account according to the assigned measurement error. The decision problem which degenerate channel to cancel and the problem how to deal with the intermediate cases do not emerge.

\subsection{Calibration}

The relative calibration factors $s_{i}$ are determined from LiI emission measurements in neutral hydrogen gas which is filled into the ASDEX Upgrade vessel after each plasma discharge. The typical $\mathrm{H}_{2} / \mathrm{D}_{2}$ gas pressure is $2 \times 10^{-4} \mathrm{mbar}<p<1.5 \times 10^{-3} \mathrm{mbar}$ measured with a baratron. The left panel of figure 3 shows a $0.3 \mathrm{~s}$ time segment of
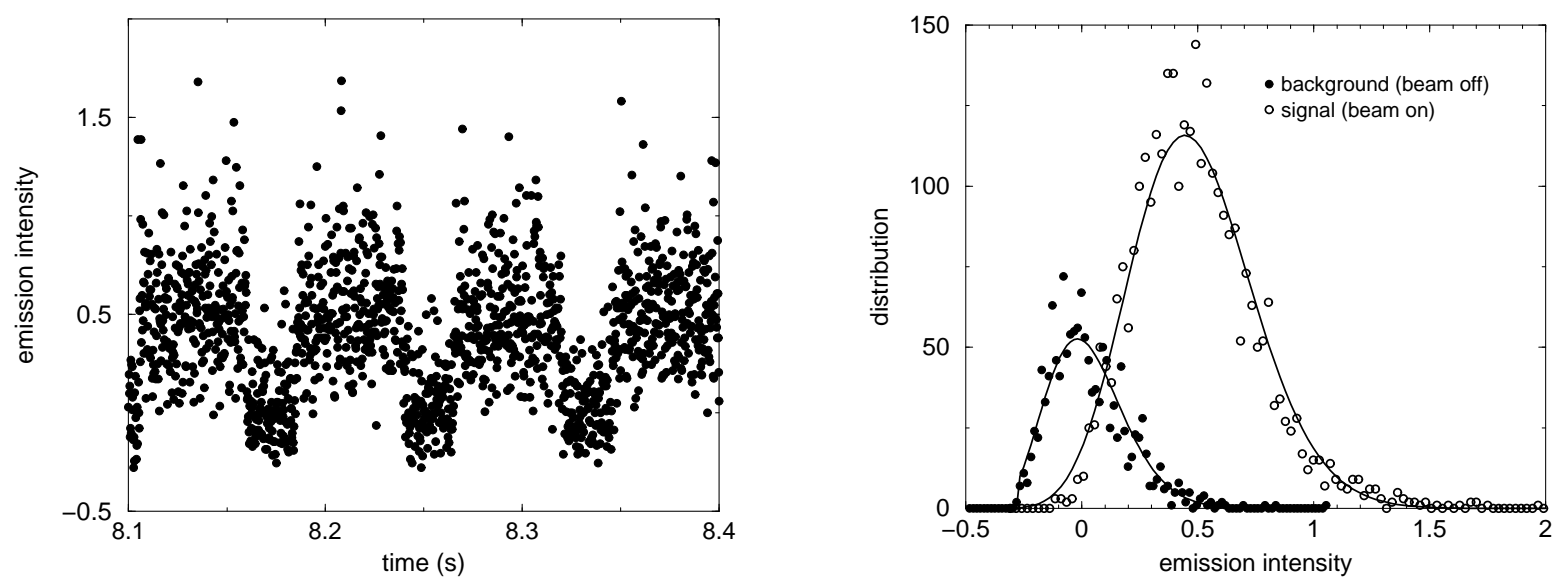

Figure 3. Left: Calibration measurement with $\mathrm{D}_{2}$ gas filling with beam on and beam off. Right: Histograms for background and signal measurement and Poisson distribution (solid lines) with estimated mean, offset and scale values.

totally $1 \mathrm{~s}$ of calibration measurement with $\mathrm{H}_{2}$ gas filling for one of the 35 lithium beam channels. The segments with beam on and off can clearly be distinguished. The smaller signal (beam off) accounts for background only and the larger signal (beam on) contains additional line emission. The background (baseline) $b_{i}$ is calculated from the mean of signals with beam off. The uncertainty of the estimated background is given by the standard uncertainty of the sample mean. 
In case of negligible beam attenuation the measured intensity profile reflects the relative radial transmissivity of the 35 spatial channels optical detection system. However, for the chosen gas pressure the neutral Li-beam is attenuated by ionization through collisions with gas molecules. The beam composition and attenuation by collisions with neutral hydrogen is quantified with a two-state rate equation model $(2 \mathrm{~s}, 2 \mathrm{p})$ with constant coefficients which can be solved analytically [10]. The atomic data are taken from [28]. The model takes into account excitation from the level $2 \mathrm{~s}$ to $2 \mathrm{p}$, ionization from $2 \mathrm{~s}$ and $2 \mathrm{p}$, and it assumes that all $\mathrm{Li}^{+}$ions are lost from the beam. This is justified by having a toroidal magnetic field in the phase of calibration approximately $1 \mathrm{~s}$ after the end of a discharge. In this phase also a spatially homogeneous gas pressure can be assumed in the torus.

Experimentally, the beam attenuation in the gas filled torus can be measured by taking the emission ratios $d_{i}\left(p_{1}\right) / d_{i}\left(p_{2}\right)$ at different gas pressures $p_{1}$ and $p_{2}$ and normalizing them to 1 for the outermost channel $i=1$. These experimental ratios turned out to be consistent with corresponding results of the analytic two-state model in the pressure range $5 \times 10^{-5}-2.5 \times 10^{-3}$ mbar. Therefore, based on this model, correction factors $\eta_{i}$ could be derived to compensate the beam attenuation effect. The correction factors $\eta_{i}$ are parameterized with a simple analytic expression

$$
\begin{aligned}
\eta_{i} & =\left(1-a z_{i}+b z_{i}^{2}\right)^{-1} \\
z_{i} & =0.441(i-1) \\
a & =c_{3} p-c_{4} p^{2}-c_{5} \\
b & =c_{1} p^{c_{2}}
\end{aligned}
$$

where $c_{1}=6.8 \times 10^{-7}, c_{2}=1.89, c_{3}=0.00105, c_{4}=8.0 \times 10^{-7}$, and $c_{5}=3.0 \times 10^{-5}$ are constants estimated from a fit to the analytic gas model of the Li-beam. The background subtracted intensities $d_{i k}-b_{i}$ at time point $k$ are multiplied with $\eta_{i}$ according to

$$
\hat{d}_{i k}=\left(d_{i k}-b_{i}\right) * \eta_{i}(p)
$$

where $d_{i k}$ is the measured intensity and $b_{i}$ is the background. $z(\mathrm{~cm})$ approximates the beam coordinate for the 35 channels $i$. The deviation of the true beam coordinates as a function of the channel index $i$ from the linear approximation provides a higher order correction and, therefore, is of minor importance for the beam attenuation. The left panel of figure 4 shows the correction factor $\eta_{i}$ for a gas pressure of $p=1.5 \times 10^{-3}$ mbar. The correction factor increases with pressure and beam coordinate $z_{i}$. During the calibration measurement the neutral gas pressure changes as depicted in the right panel of figure 4 for channel $i=35$ where the attenuation effect is largest. Therefore, the correction factor depends on the beam coordinate as well as on time.

The obtained calibration profile contains the radial dependence of all calibration quantities [4]:

$$
s_{i}=\frac{1}{N} \sum_{k}^{N} \hat{d}_{i k}
$$



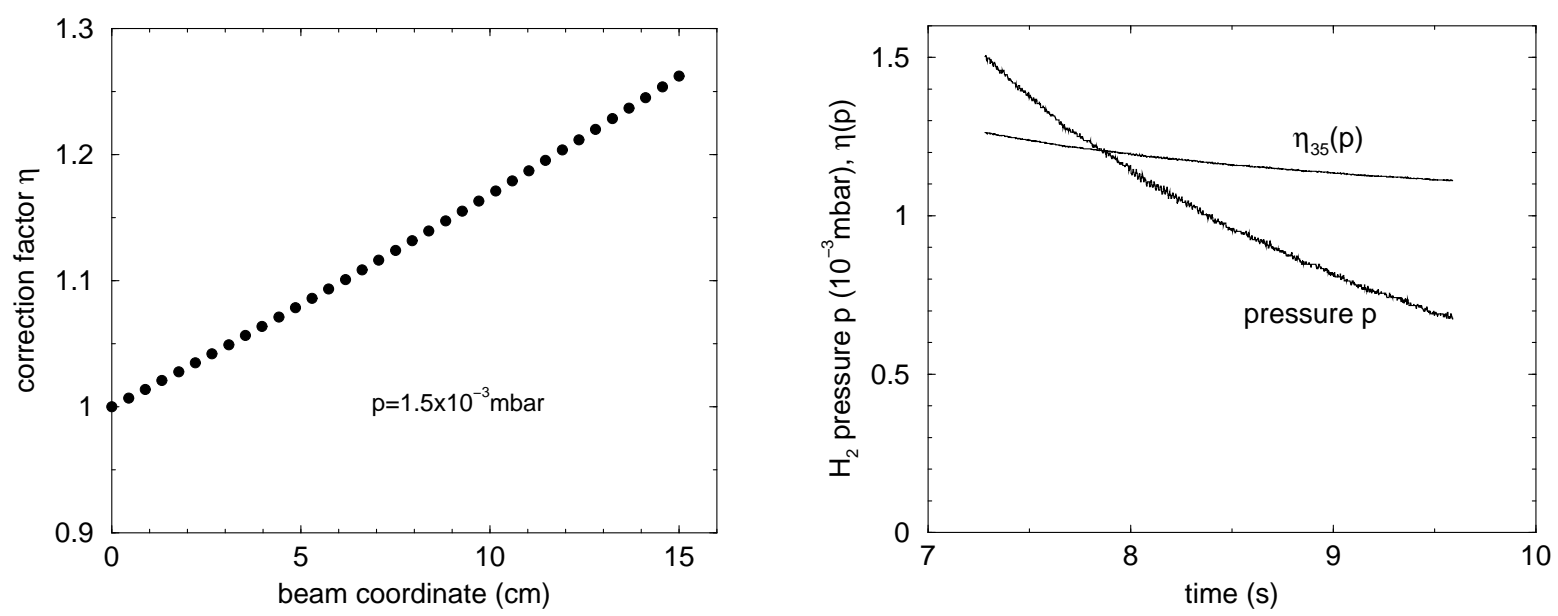

Figure 4. Left: Correction factor $\eta$ as a function of the beam coordinate $z_{i}(\mathrm{~cm})$ for an hydrogen pressure $p=1.5 \times 10^{-3}$ mbar. Right: Pressure $p$ during calibration measurement and pressure correction factor $\eta_{i}$ due to beam attenuation (here for spatial channel $i=35$ )

The variance $\Delta s_{i}^{2}$ of the calibration constant $s_{i}$ is given by the variance of the mean of the compensated calibration measurement $\hat{d}_{i k}$ over all time points of the calibration measurement plus the variance of the background uncertainty. The uncertainty of the pressure measurement is $1 \times 10^{-5}$ mbar which is about $1 \%$ of the calibration pressure. The corresponding distortion of the estimated density profile due to the uncertainties on $s_{i}$ induced by the pressure uncertainty is, therefore, negligible. The uncertainty in $s_{i}$ results in a further modification of the $\chi^{2}$-term in the likelihood [17]:

$$
\chi^{2}=\sum_{i j}^{N_{d}}\left[\frac{\left(d_{i j}-d_{i j, \mathrm{~b}}-D_{i}\left(n_{\mathrm{e}}\right)\right)^{2}}{\sigma_{i j}^{2}+\sigma_{i j, \mathrm{~b}}^{2}+\left(D_{i}\left(n_{\mathrm{e}}\right) \Delta s_{i} / s_{i}\right)^{2}}\right]
$$

This completes the likelihood.

\subsection{Prior}

The prior pdf quantifies the information we have about the parameters of interest independent on the measured data. In the present analysis, we assume that the density profile is mostly monotonically decreasing from the plasma center to the edge. To reduce unphysical density values or unreasonable profile oscillations weak monotonicity conditions are applied. A weak monotonicity condition penalizes profile segments decreasing with the beam coordinate $z$ (from outside to inside) using a proper regularization parameter whereas monotonically increasing profile segments are not affected. Since we do not want to exclude hollow profiles completely, we introduce a Gaussian penalization term on non-monotonic density profiles

$$
p\left(\vec{n}_{e}\right) \propto\left(\begin{array}{c}
\exp \left\{-\sum_{i=1}^{N-1} \frac{\left(n_{e, i+1}-n_{e, i}\right)^{2}}{\left(n_{e, i+1}+n_{e, i}\right)^{2} s_{g}^{2}}\right\} \text { for } n_{e, i+1}<n_{e, i} \\
1 \quad, \quad \text { elsewhere }
\end{array}\right)
$$


where $s_{g}$ provides a regularization parameter which quantifies how much we believe in monotonic profiles. This penalization term mainly acts as a weak constraint to the space of density profiles consistent with physical considerations. If the measured data can only be fitted consistently with non-monotonic profiles we can relax the penalization by increasing $s_{g}$. Then, we have to check if the non-monotonic behavior is due to a real physical effect, or if there is a systematic uncertainty in the data or in the modeling we have to resolve.

In the present work, the density profile is parameterized by cubic spline polynomials. Since density values have to be positive, a natural representation of density profiles is given by the exponential of cubic spline polynomials. Any other functional description of $n_{\mathrm{e}}$ can be used too as far as the complete space of physically reasonable $n_{\mathrm{e}}$ profiles is covered. Cubic splines are well suited for providing a reasonable balance between flexibility and robustness against insignificant structures. We have also used Hermite polynomials which have the advantage that monotonicity constraints can be easily employed but Hermite polynomials frequently show structures and edges where there is no evidence in the data.

A second prior pdf used penalizes density profile curvature as it is applicable for a cubic spline representation. Details can be found in [29]. The regularization parameter weighting the curvature term was chosen such that the curvature term provides a smooth profile only to avoid noise fitting. Since the data noise is absolutely calibrated we can recognize noise fitting by recording the $\chi^{2}$-misfit.

\section{Implementation and numerical effort}

The numerical effort of the new probabilistic analysis is comparable to the conventional analysis for a single time slice. The conventional method has to iterate for a consistent calibration constant $\alpha$ whereas the probabilistic method includes an optimization step to find the density profile and parameter $\alpha$ which fits the data best. For a typical set of 14 parameters (13 spline parameters plus $\alpha$ ) the optimization runs within a couple of seconds and the determination of the profile uncertainty another $\approx 1 \mathrm{~s}(3 \mathrm{GHz}$ Linux PC). The most time consuming part of the analysis is given by the solver of the system of coupled linear differential equations. An analysis on an inter-shot basis (30 min) would therefore allow for about 500 time slices to be analyzed on a single processor. Assuming a measurement time of $5 \mathrm{~s}$ one would obtain a density profile each $10 \mathrm{~ms}$ which is the typical resolution obtained with the conventional approach. Routine analysis with the new approach was done on a linux cluster with 14 dual processor boards employing Hyper-Threading. This allows to run 56 time slices in parallel which increases the temporal resolution for density profiles to about $200 \mu \mathrm{s}$. The maximum possible temporal resolution of $50 \mu$ s either runs for about 2 hours or the analysis is restricted to a selection of 1-2 s discharge time for an inter-shot analysis.

The computational effort increases both, with the size of the parameter set and with the size of the data set. For a combined analysis of lithium beam data and 
interferometry data the number of data as well as the number of parameters increase. The size of the parameter set increases because this combination allows to determine full density profiles. Actually, the combination of lithium beam and DCN interferometry data at ASDEX Upgrade increases computation time for a density profile per time slice by about one order of magnitude. A further increase in computation time arises if additional diagnostics are added such as Thomson scattering and ECE for simultaneous estimation of electron density and temperatures profiles.

\section{Comparison of results from conventional and probabilistic approach}
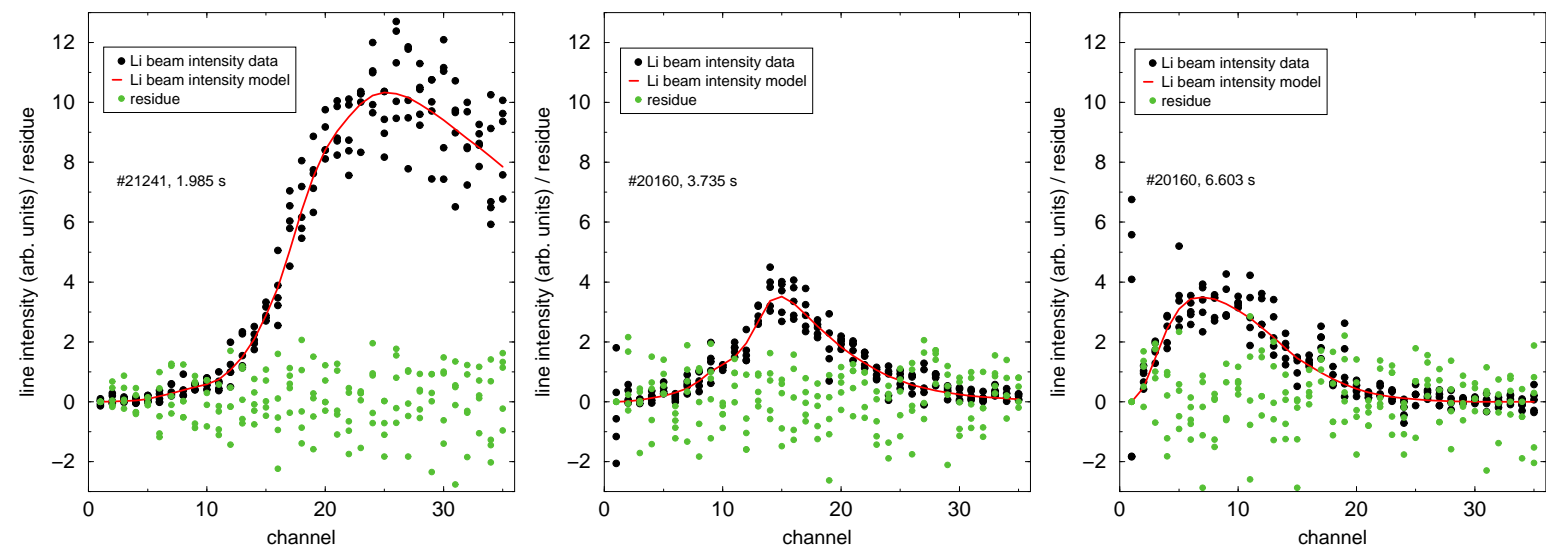

Figure 5. Data, fitted emission intensities and residuals for 3 plasma scenarios

To demonstrate the advanced data analysis method, an ohmic discharge with low (\#21241, 2s), and an H-mode discharge with medium (\#20160, 3.8s) and high (\#20160, 6.6s) density regimes at ASDEX Upgrade were chosen. Figure 5 shows the line emission intensity profiles, the fitted emission curves and the residuals of the misfit of the data and the model weighted with the data uncertainties. Stationary plasma conditions were chosen to allow a simultaneous fit of a single density profile to 5 neighboring time frames $200 \mu \mathrm{s}$ apart. The residuals show the thorough description of all measurement uncertainties. The residuals would easily reveal non-stationary conditions where density profiles must be fitted for individual time frames because $n_{\mathrm{e}}$ changes within the time window chosen.

Figures 6, 7 and 10 show a comparison of the density profiles as a function of $\rho_{\text {pol }}$ obtained with the new improved analysis tool, the classical IPP algorithm and the (core or edge) Thomson scattering diagnostics (shifted with $\Delta \rho_{\text {pol }}=0.01$ ). The left panel of the figures show all profiles within a time frame of $0.2 \mathrm{~s}$ and the right panels depict single density profiles including estimation uncertainties.

The old analysis tool does not allow to obtain density profiles for small densities because the inner boundary condition cannot be fulfilled as is the case for figure 6 . The new probabilistic data analysis tool allows to obtain density profiles for any density 

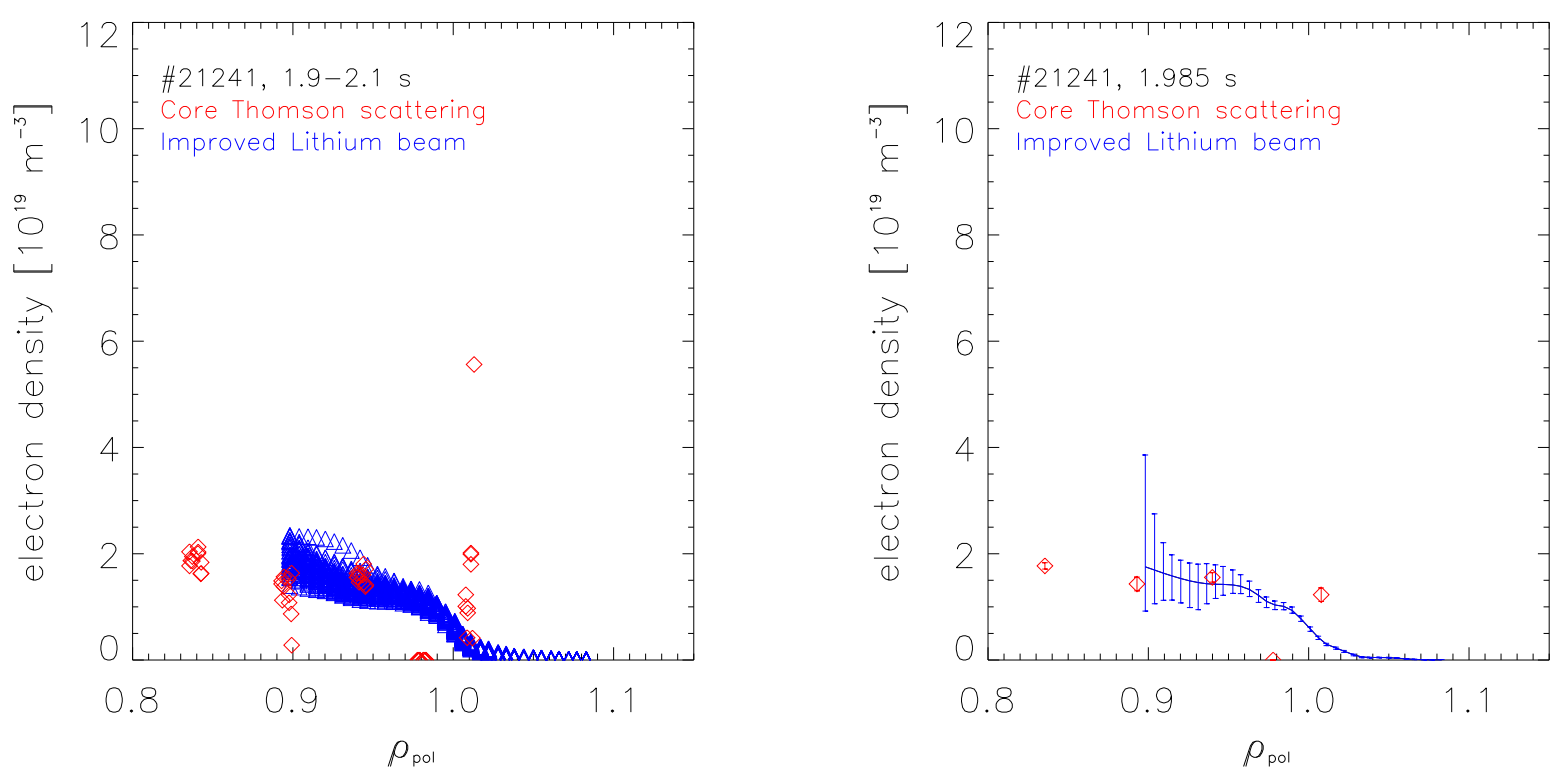

Figure 6. Comparison of the density profiles (\#21241) obtained with the new analysis tool (blue triangles) and the core Thomson scattering diagnostics (red diamonds) Left: series of density profiles (1.9s-2.1s) Right: profile and estimation uncertainties for a single time frame

regime independent of the existence of boundary or singularity conditions. From the error bars and the scatter of the profiles one can conclude that the reliability of the profile reconstruction for the small density regime is large up to $\rho_{\mathrm{pol}}>0.92$. This is not because the Li-beam becomes too faint for $\rho_{\text {pol }}<0.92$ but due to the position of the innermost spatial channel. Extending the spatial channels into the plasma would not only allow to recover the density profile for smaller $\rho_{\text {pol }}$ values but, additionally, would increase the estimation precision of $n_{\mathrm{e}}$ of the present channels due to the non-locality of the emission profile.

Figure 7 depicts an H-mode discharge with medium electron density. The pedestal is well resolved for the edge Thomson system and the new Li-beam analysis tool whereas the old tool does not provide the level of the pedestal top. Again, the reliability of the profile reconstruction for the medium density regime is large for $\rho_{\text {pol }}>0.93$. The error bars become large for $\rho_{\text {pol }}<0.93$ showing that the information content in the data about this part of the density profile diminishes. The increase of the error bars originate from the decrease of the beam intensity. The left panel of figure 8 shows a density profile and its confidence interval as a function of the beam axis and the right panel depicts the corresponding occupation densities of the 9 energy levels of neutral lithium (dot-dashed $2 \mathrm{~s}$, dashed $2 \mathrm{p}, \ldots$ ), the neutral beam intensity (solid line), and the electron-impact population coefficient of the $2 \mathrm{p}$ energy level (dotted). The beam intensity is proportional to the sum of the 9 energy levels of neutral lithium. The zero point of the electronimpact population coefficient of the 2 p energy level $\left(\sum_{j=1}^{N_{\mathrm{Li}}} a_{2 j}\left(T_{\mathrm{e}}(z)\right) N_{j}(z)\right.$; see equation 1 ) constitutes the singularity condition of the conventional analysis technique where 

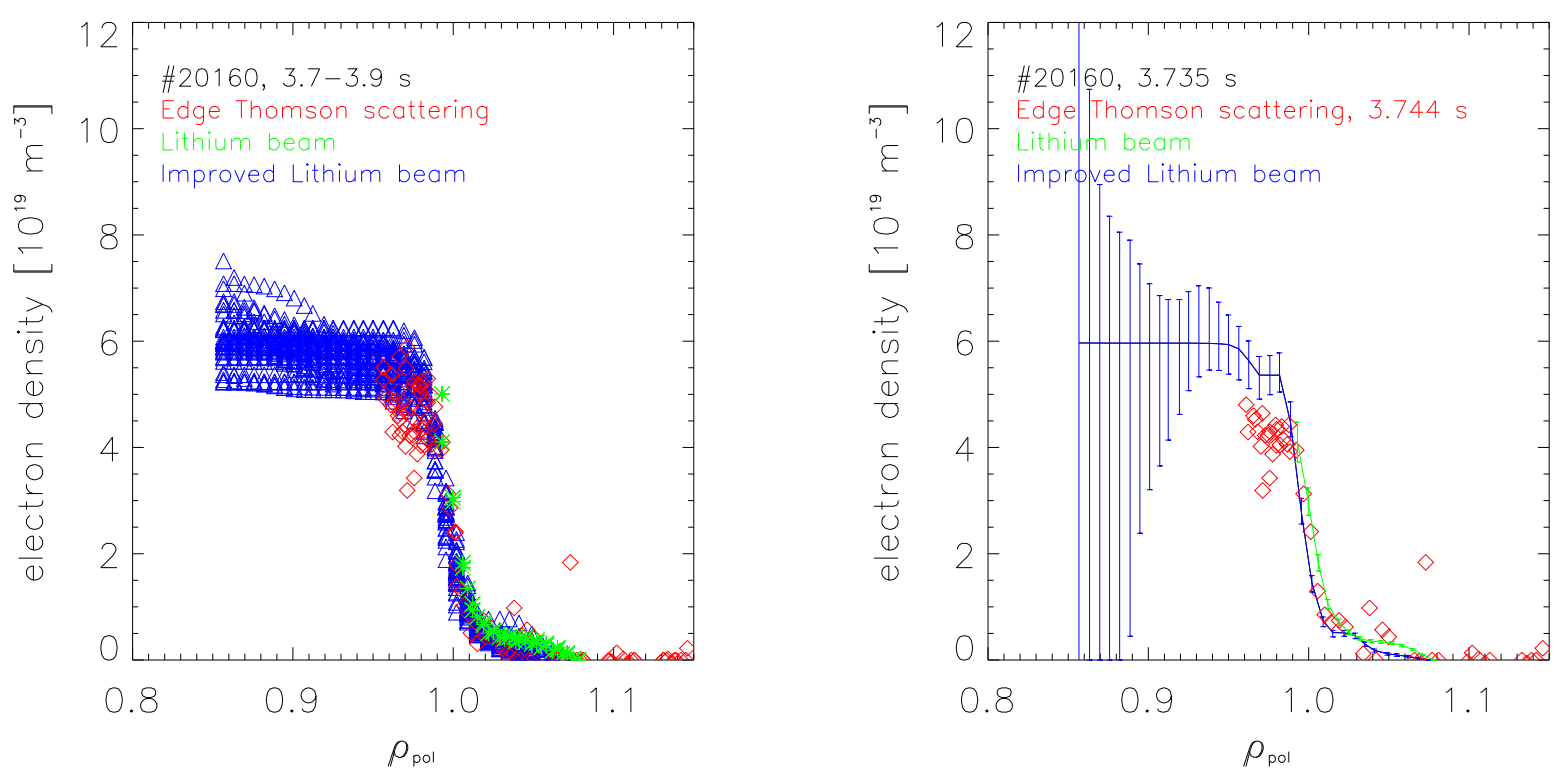

Figure 7. Comparison of the density profiles (\#20160) obtained with the new analysis tool (blue triangles), the classical IPP algorithm (green stars) and the edge Thomson scattering diagnostics (red diamonds) Left: series of density profiles (3.7s-3.9s) Right: profile and estimation uncertainties for a single time frame
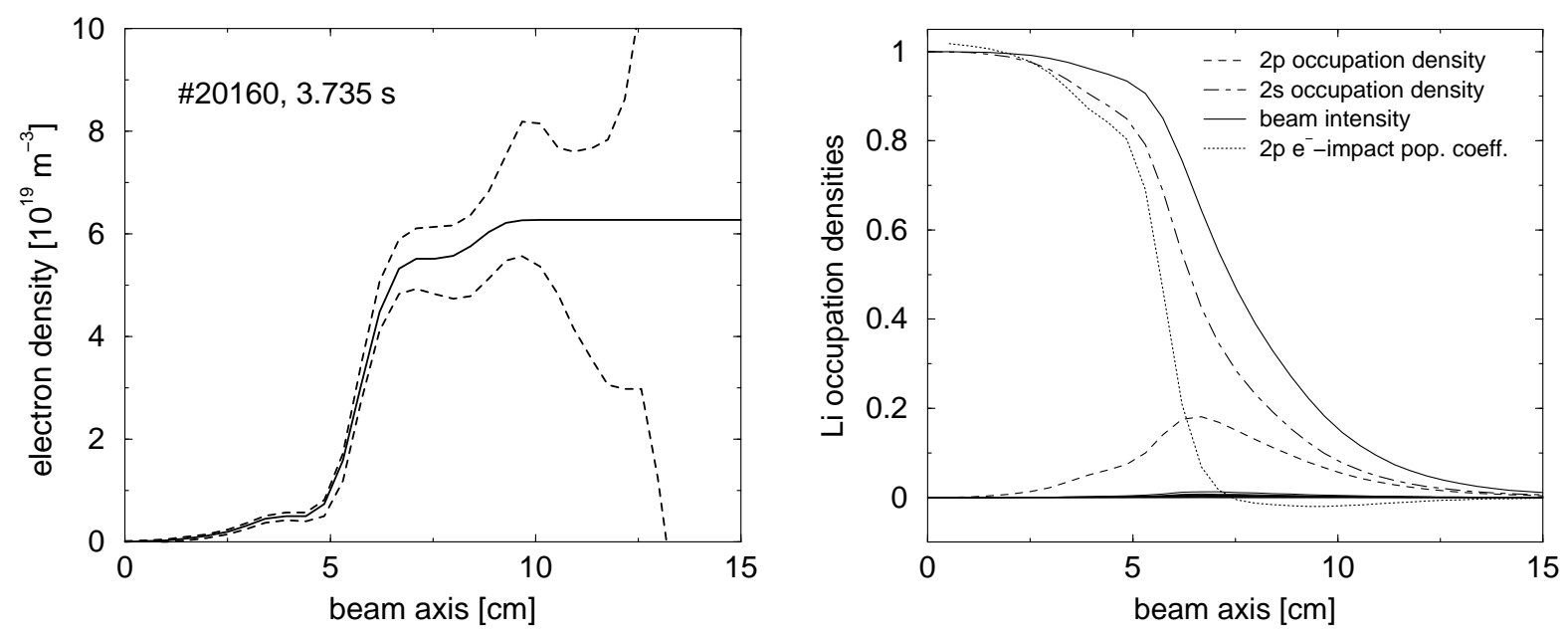

Figure 8. Left: Density profile and confidence interval as a function of the beam axis. Right: occupation densities of the 9 energy levels of neutral lithium, the neutral beam intensity, and the electron impact population coefficient of the $2 p$ energy level.

electron impact population and de-population cancel. At the singularity point (here at about $z_{s}=7.4 \mathrm{~cm}$ ) the explicit dependence on the density disappears. Nevertheless, the confidence interval of $n_{\mathrm{e}}$ (right panel) does not increase significantly at this point showing that the increase of the profile uncertainty with increasing beam coordinate is not due to this singularity point but due to the decrease of the beam intensity. The explicit singularity point has a minor effect on the error bar of $n_{\mathrm{e}}$ because the density 
at this point is determined not only from the line emission at this point but from the full emission profile after that point $\left(z>z_{s}\right)$.
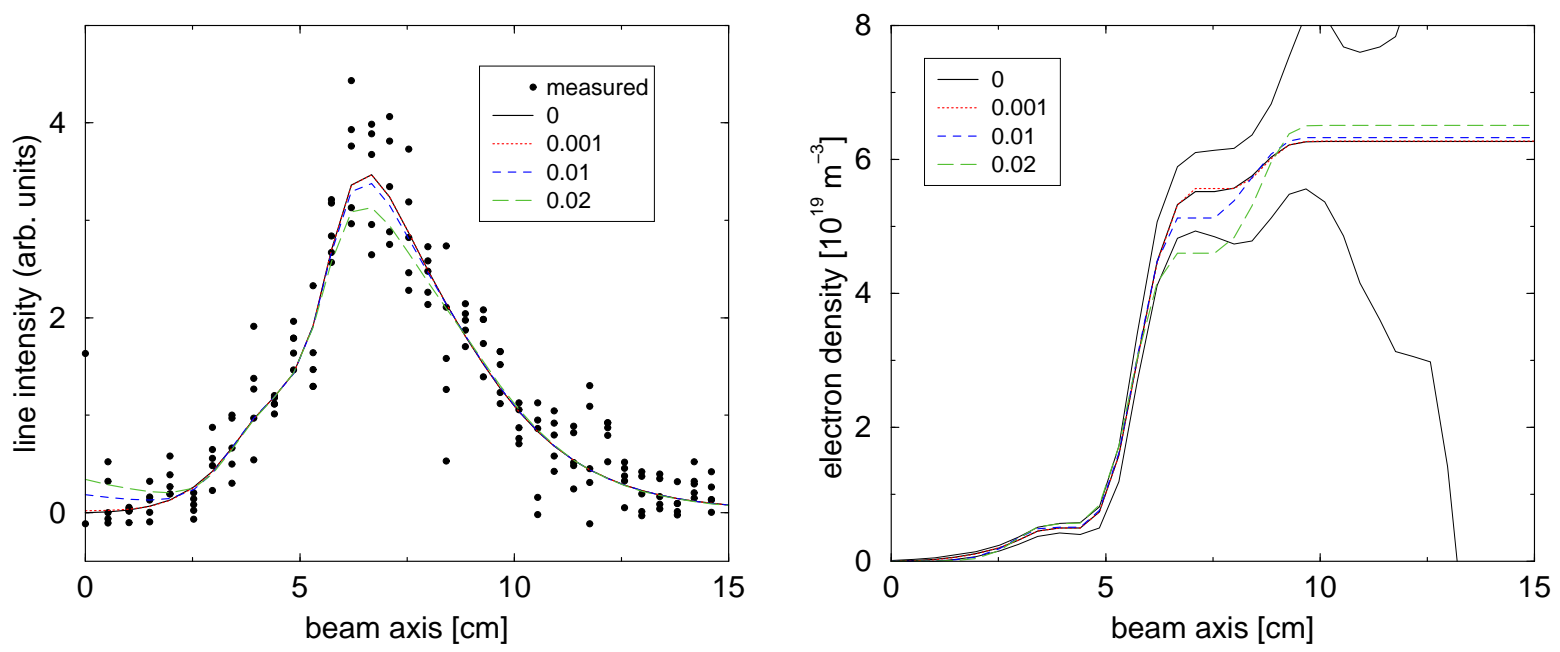

Figure 9. Left: 2 p occupation densities $N_{2}(z)$ (multiplied with $\alpha$ ) as a function of the beam axis for four different initial boundary conditions $\left(N_{2}(z=0)=\right.$ $0,0.001,0.01,0.02)$ at the plasma entrance. Right: Corresponding density profiles.

Another prominent difference between the conventional and the probabilistic data analysis approach is given by the different sensitivity of the density profile on changes of the boundary condition at $z=0$ (equation 2). It is known for a long time that the conventional approach produces profiles which very sensitively depend on this initial boundary condition of the $2 \mathrm{p}$ occupation density $N_{2}(z=0)$ if $\alpha$ is not adaptively chosen. The reason is a close interdependency between this boundary condition and the absolute calibration constant $\alpha$ which is determined with a shooting method known for its numerical instability. The new probabilistic method treats $\alpha$ as a fitting parameter with an estimated relative uncertainty of typically about $3 \%$. Therefore, the density profiles obtained with the probabilistic method do not depend sensitively on the choice of $\alpha$ within its error bars. As a consequence of the robustness of the estimated profiles on $\alpha$, it was not expected that the profiles depend sensitively on the initial boundary condition. Figure 9 shows on the left panel a measured line emission profile (dots) and the emission profiles simulated with different initial boundary conditions $N_{2}(z=0)=0,0.001,0.01,0.02$ and on the right panel the corresponding density profiles. For this data set the boundary conditions with $N_{2}(z=0)=0,0.001$ are consistent with the data and the respective density profiles are indistinguishable. For $N_{2}(z=0)=0.01,0.02$ changes are observed in the simulated emission profiles and in the density profiles in the order of the profile uncertainties. The important fact is that for these increased occupation densities the misfit between the measured and the simulated data is significantly increased by multiples of the standard deviation. Therefore, such large boundary occupation densities can be ruled out by the data or, as a consequence, the data allow to include the initial boundary condition into the list of parameters to 
be fitted. To estimate $N_{2}(z=0)$ from the data provides a major advantage of the probabilistic method because this initial boundary condition depends in addition to any plasma for $z<0$ also on the neutral gas density between the lithium beam generation box and the plasma entrance. Since the distance between the lithium beam generation box and the plasma entrance is in the order of meters and the neutral gas density might suffer from rapid changes, a robust and automatic time-resolved estimation technique of both, $\alpha$ and $N_{2}(z=0)$, is highly required.

The nuisance parameters $\alpha$ and $N_{2}(z=0)$ can, therefore, be fully included into the framework of the probabilistic approach. If one is not interested in the actual values of those parameters but on the density profile only, the probabilistic approach requires to marginalize (integrate out) these parameters. As a consequence of the marginalization, the uncertainties of $\alpha$ and $N_{2}(z=0)$ propagate into the uncertainties of the quantity of interest $n_{\mathrm{e}}$. Since marginalization of nuisance parameters is numerically rather expensive, for a routine analysis with a temporal resolution of up to $50 \mu \mathrm{s}$ a pragmatic treatment of the nuisance parameters as fitting parameters with Gaussian error propagation is reasonable and sufficient.
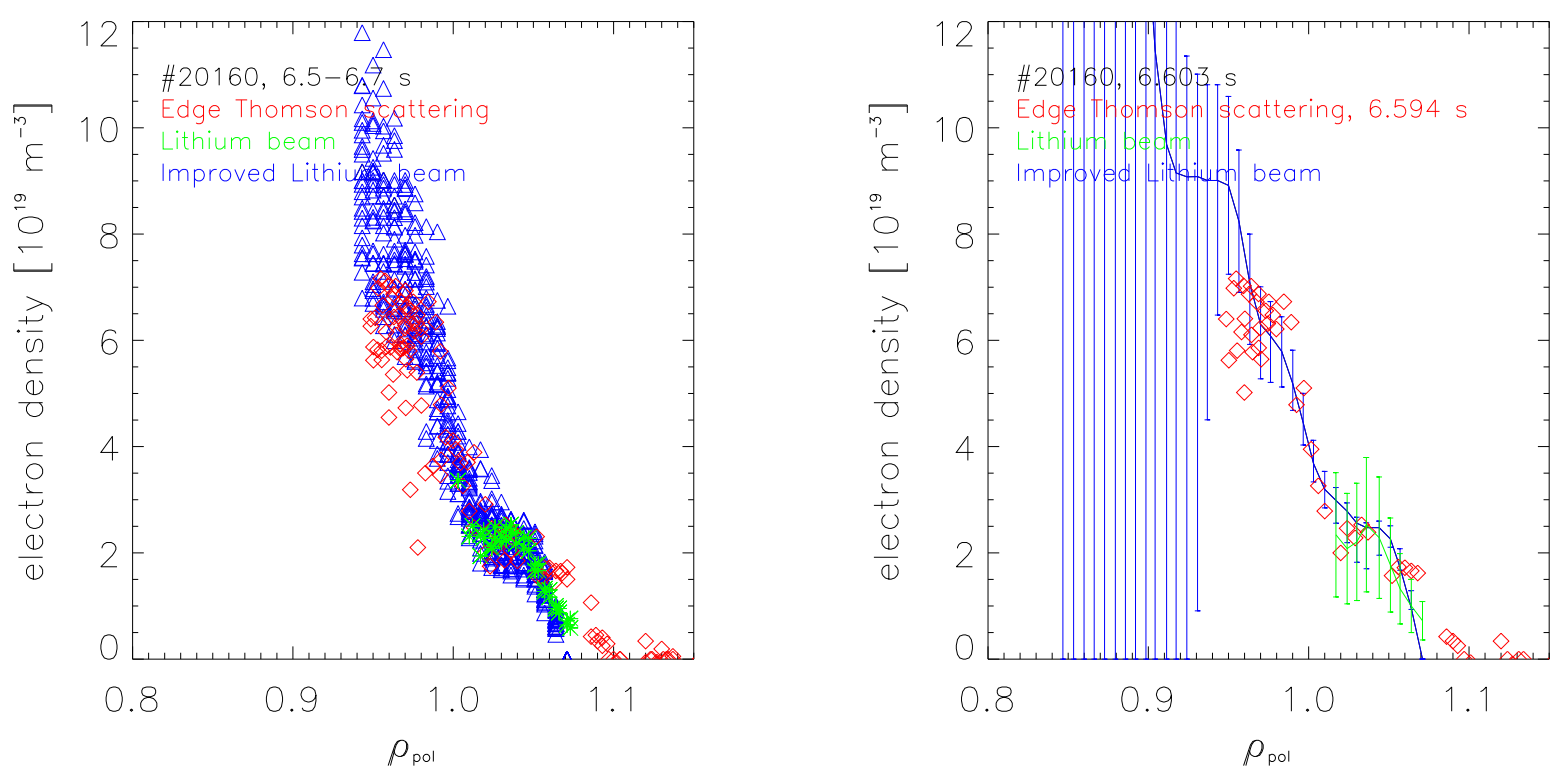

Figure 10. Comparison of the density profiles (\#20160) obtained with the new analysis tool (blue triangles), the classical IPP algorithm (green stars) and the edge Thomson scattering diagnostics (red diamonds) Left: series of density profiles (6.5s6.7s) Right: profile and estimation uncertainties for a single time frame

A high-density regime within the same discharge (\#20160, 6.5-6.7 s) is depicted in figure 10. The old density evaluation stops in the SOL whereas the new tool allows to reach the pedestal top although the level of the pedestal top is not clearly resolved. This is due to the diminishing Li-beam and can be resolved only with larger beam energy. The evaluated profile is reliable for $\rho_{\text {pol }}>0.96$. Both the position of the pedestal as well as the position of the limiter shadow can be resolved. 


\section{Temporal resolution}

With the conventional analysis tool temporal binning is necessary to obtain the required signal-to-noise ratio. ELM-resolved electron densities are obtained with temporal binning relative to the onset time of the ELMs assuming that the ELMs are comparable. Due to the consistent error treatment of the new probabilistic tool single emission profiles can be analyzed with the maximum sampling frequency of $20 \mathrm{kHz}$. Therefore, binning of the ELMs relative to their onset times is no longer necessary.

To depict the newly achieved temporal resolution of $50 \mu$ s an H-mode discharge (\#22561) was chosen including a type I ELM. The left panel of figure 11 shows the
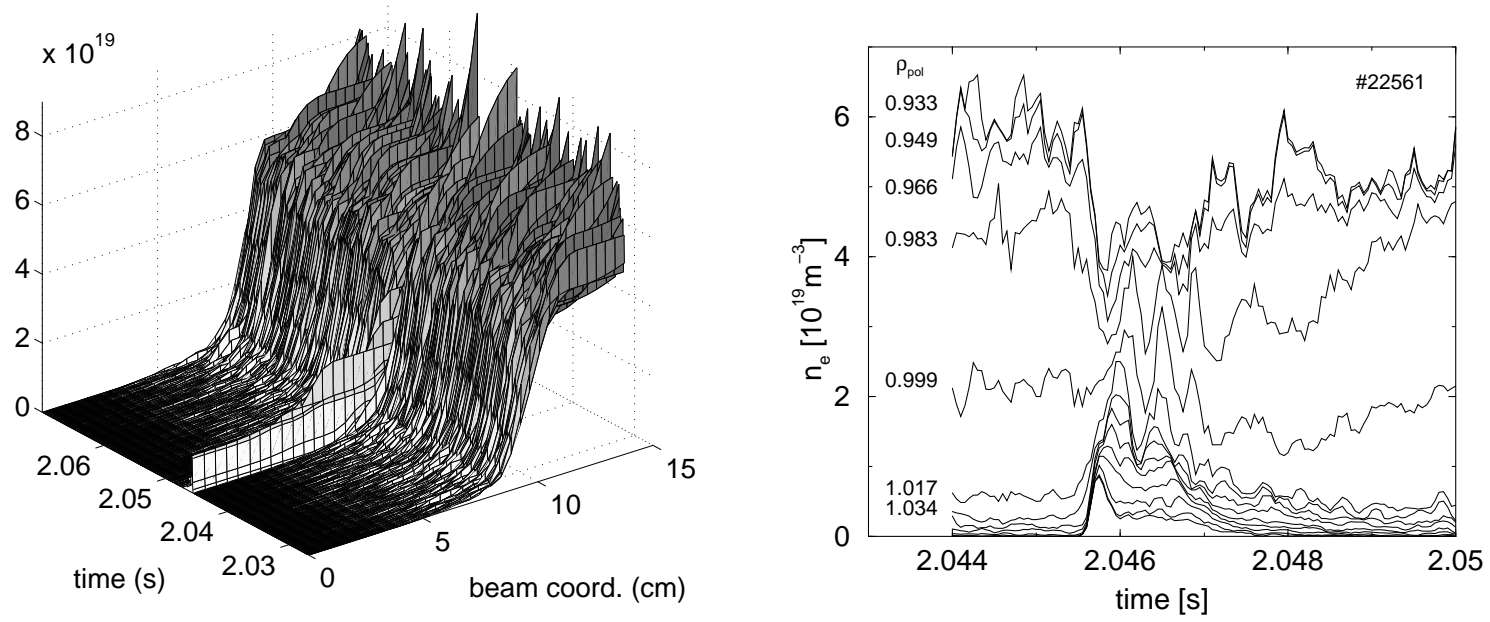

Figure 11. Left: Density profiles as a function of the beam coordinate and time (\#22561). The temporal resolution is $50 \mu \mathrm{s}$. After $t=2.0455 \mathrm{~s}$ a type I ELM appears. Right: Density profiles for the same shot as a function of $\rho_{p o l}$ and time. Close to the separatrix $\left(\rho_{\text {pol }}=0.999\right.$ and $\left.\rho_{\text {pol }}=0.983\right)$ oscillations with a time period of about $400 \mu$ s are clearly resolved.

temporal evolution of the density profile as a function of the beam coordinate from $t=2.025-2.070 \mathrm{~s}$. The temporal resolution is $\Delta t=50 \mu \mathrm{s}$. At about $t=2.0455 \mathrm{~s}$ a type I ELM appears. The right panel shows the temporal evolution of the density profile as a function of $\rho_{\text {pol }}$ from $t=2.044-2.050 \mathrm{~s}$. For reasons of presentation only each second spatial channel is shown. Close to the separatrix $\left(\rho_{\text {pol }}=0.999\right.$ and $\left.\rho_{\text {pol }}=0.983\right)$ oscillations with a time period of about $400 \mu$ s are clearly resolved. To reduce statistical fluctuations three neighboring profiles were averaged.

Figure 12 shows 4 single density profiles including upper and lower confidence bands. The leftmost profile is for a time point at $t=2.04540 \mathrm{~s}, \Delta t_{\mathrm{ELM}}=-150 \mu \mathrm{s}$ before the ELM onset, and the other profiles are at different time points during the ELM at $2.04575 \mathrm{~s}$ $\left(\Delta t_{\mathrm{ELM}}=250 \mu \mathrm{s}\right), 2.04600 \mathrm{~s}\left(\Delta t_{\mathrm{ELM}}=500 \mu \mathrm{s}\right)$, and $2.04625 \mathrm{~s}\left(\Delta t_{\mathrm{ELM}}=750 \mu \mathrm{s}\right)$. The rightmost profile at $2.04625 \mathrm{~s}$ is not recovering the full density prior to the ELM because this occurs at a much later time point as can be seen in figure 11. Please note that 

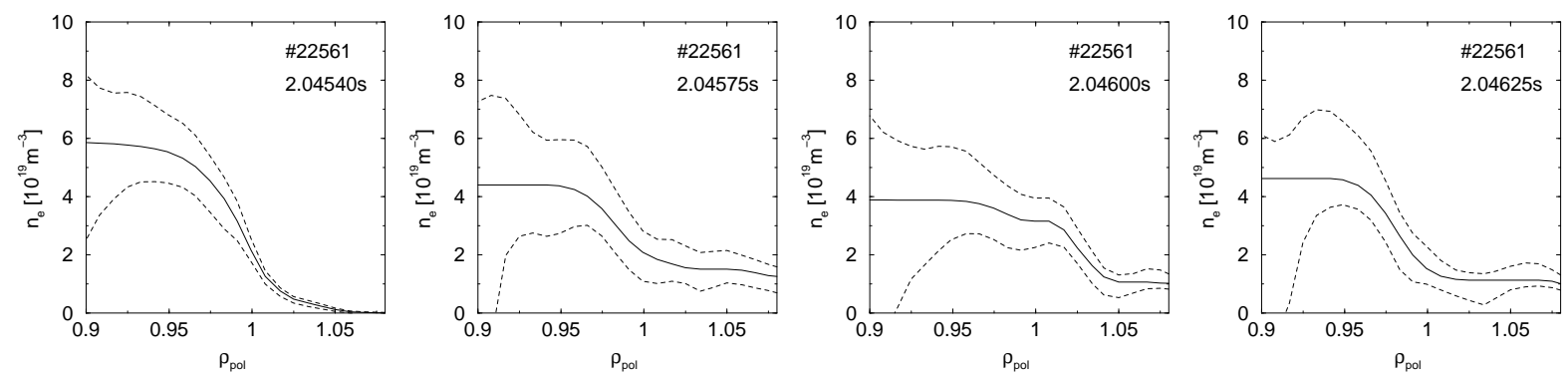

Figure 12. Density profiles at $2.04540 \mathrm{~s}\left(\Delta t_{\mathrm{ELM}}=-150 \mu \mathrm{s}\right), 2.04575 \mathrm{~s}\left(\Delta t_{\mathrm{ELM}}=\right.$ $250 \mu \mathrm{s}), 2.04600 \mathrm{~s}\left(\Delta t_{\mathrm{ELM}}=500 \mu \mathrm{s}\right)$, and $2.04625 \mathrm{~s}\left(\Delta t_{\mathrm{ELM}}=750 \mu \mathrm{s}\right)$. The temporal resolution is $50 \mu \mathrm{s}$.

the mapping from the beam axis to the $\rho_{\text {pol }}$-axis is taken from a single equilibrium calculation for a time point prior to the ELM. Therefore, the actual position of the separatrix position during the ELM most probably is not correct and the profile has to be shifted. A refined equilibrium calculation will be subject of continuing work.
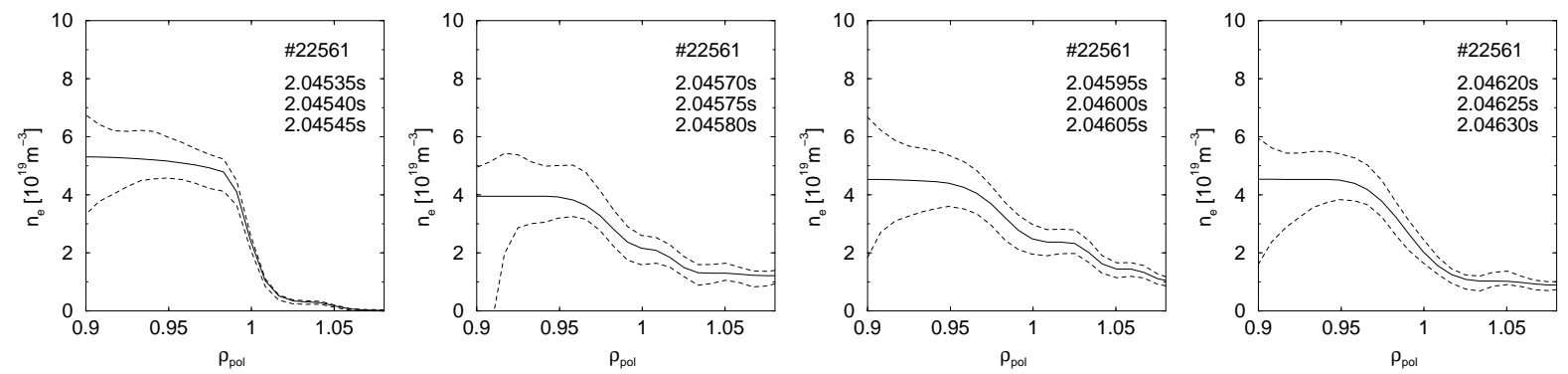

Figure 13. Same as for figure 12 but each density profile estimated jointly from 3 consecutively measured emission profiles (temporal resolution of $150 \mu \mathrm{s}$ ).

Analogous to figure 12, figure 13 shows density profiles where each profile is estimated jointly from 3 consecutively measured emission profiles (temporal resolution of $150 \mu \mathrm{s}$ ). The profile uncertainties decrease and the edge barrier is resolved more clearly at $t=2.04540 \mathrm{~s}$.

A detailed analysis of the development of the density profiles during an ELM crash is beyond the scope of this paper and will be topic of subsequent work.

\section{Dependence on temperature and impurity content}

The dependence of the estimated density profile on the electron temperature is expected to be small above the ionization threshold of lithium at $5.4 \mathrm{eV}$. Small changes in the energies below the ionization threshold can result in significant changes in the occupation densities of the excited states with $n \geq 3$. Therefore, analysis of lowtemperature plasmas with lithium beams require precise knowledge about the electron temperature. For temperatures above the ionization threshold it was observed that the 
effect of changes in the temperature on the density profile is small using the most recent data base [14]. Figure 14 depicts the dependence of the estimated density profile (left
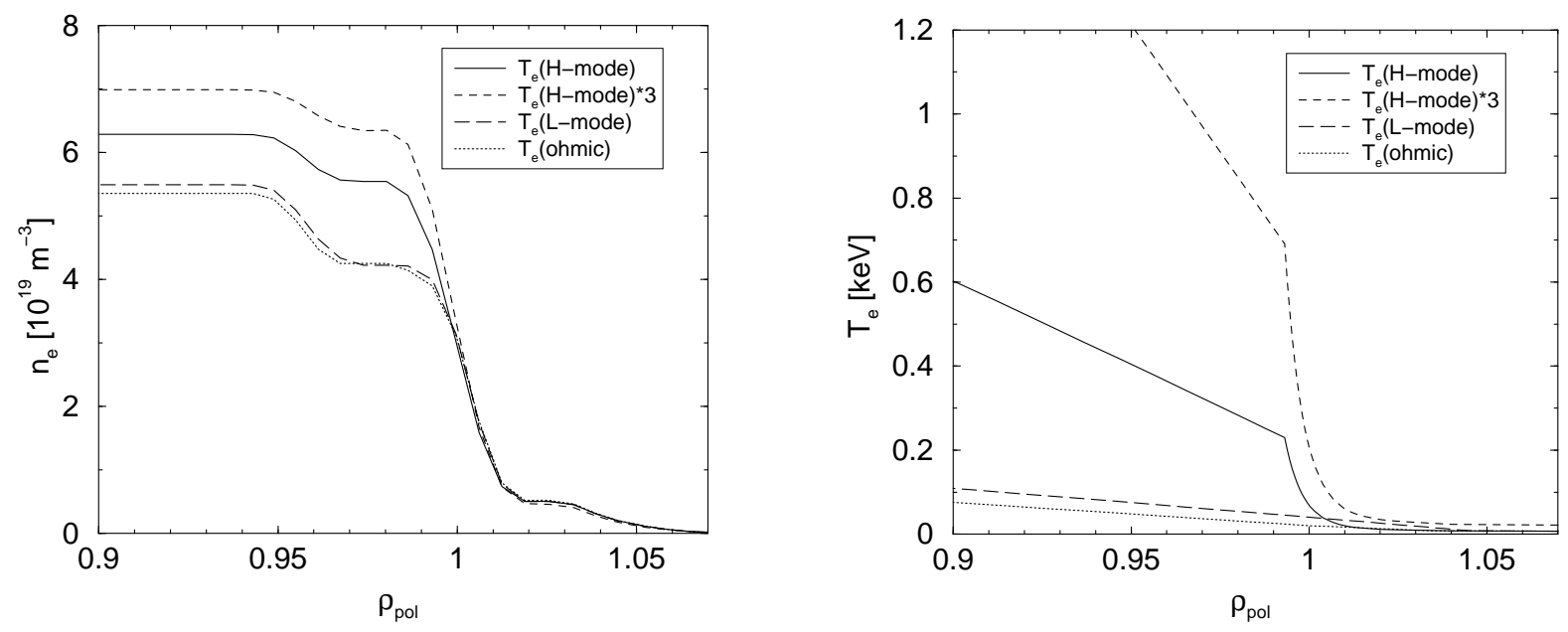

Figure 14. Density profiles (\#20160, 3.735 s) (left panel) for different choices of temperature profiles (right panel).

panel) on different choices of the temperature profile (right panel) for the shot and time slice as shown in figure 7 (right). Typical temperature profiles for H-mode $(2 \mathrm{x})$, Lmode and $\mathrm{OH}$-mode were chosen. Though an unphysically large range of temperature regimes is covered, the effect on the density profile is small compared or at most in the order of the uncertainty of $n_{\mathrm{e}}$. The differences in $n_{\mathrm{e}}$ for $\rho_{\mathrm{pol}}<1$ seem to be large but they are, even for these large differences in temperature, in the order of the uncertainty of $n_{\mathrm{e}}$ for this H-mode scenario. Actually, when the density profile is kept constant and only the temperature is changed within reasonable values, the variation in the overall deviance $\left(\chi^{2}\right)$ is small $(<1)$, which provides a statistical argument for the weak influence of the temperature on the reconstructed density profile. Additionally, the change in the deviance is very small $(\ll 1)$ for optimized density profiles using different temperature profiles. This confirms that a simultaneous estimation of density and temperature profiles from lithium beam data only is not feasible.

For routine analysis of density profiles at ASDEX Upgrade an automatic regime identification and corresponding choice of typical temperature profiles was established, because edge temperature profiles are not routinely provided. A regime identification method in ASDEX Upgrade [30] is combined with a comparison of the total and ohmic heating power to distinguish H-mode, L-mode and ohmic phases for each time slice.

The dependence of the estimated density profiles on the impurity concentration is negligible. Figure 15 depicts density profiles for a wide range of $Z_{\text {eff }}$ values assuming constant averaged charge $q=4$ for a beam energy of $E_{\text {beam }}=40 \mathrm{keV}$. The changes in the fitted profiles are much smaller than the estimation uncertainty shown in figure 7 . 


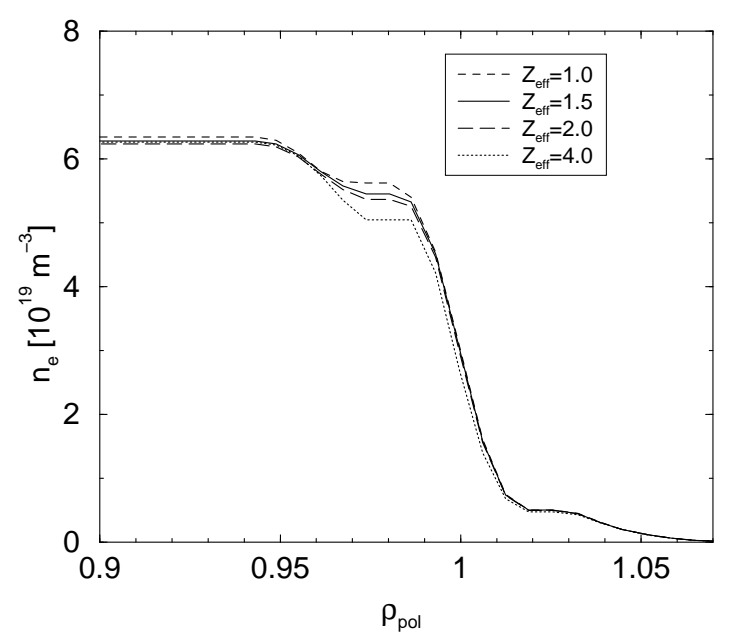

Figure 15. Density profiles (\#20160, $3.735 \mathrm{~s}$ ) for different impurity concentrations $\left(Z_{\text {eff }}\right)$.

\section{Regularization parameters}

The combined analysis of measured data and prior information in the framework of Bayesian probability theory is straightforward to be performed. If the prior information, e.g. profile monotonicity or curvature penalization, is not clearly given, a regularization parameter can be introduced which provides a relative weight between the measured data and the prior information. In the probabilistic framework, hyper-prior pdfs have to be set on the regularization parameters, as any other nuisance parameters, which describe what we know or do not know about these parameters. Then, the regularization parameters have to be marginalized to propagate their uncertainties to the uncertainties of the parameters of interest $\left(n_{\mathrm{e}}\right)$ [31]. Since marginalization of nuisance parameters is numerically rather expensive, a pragmatic approach have to be employed for any time-critical analysis. A frequently used, easy and fast approach is given by selecting reasonable values for the regularization parameters. An expedient criterion for selecting their values is given by the misfit of the measured and modeled data. The optimal misfit should account only for the uncertainty in the measured data but should not contain significant structures in the data beyond the data error bars.

The left panel of figure 16 shows density profiles obtained with 5 different values for the regularization parameter $s_{g}$ penalizing deviations from monotonicity (equation $25)$. The routinely used value is $s_{g}=1(\times 0.001)$ for which also the profile error bars are shown. The monotonicity constraint becomes weaker for increasing values of $s_{g}$. The profiles for $s_{g}=0.1,1,10$ have a high degree of monotonicity and are hardly to be separated. The right panel depicts the corresponding $\chi^{2}$-misfit values. Up to a value of $s_{g}=30$ the decrease of the $\chi^{2}$-misfit with the increase of $s_{g}$ is small. For $s_{g}>30$ the misfit decreases rapidly with $s_{g}$ because insignificant structures in the data, structures within the error bars, are fitted. On the basis of a solid error assessment, the misfit $\chi^{2}$ 

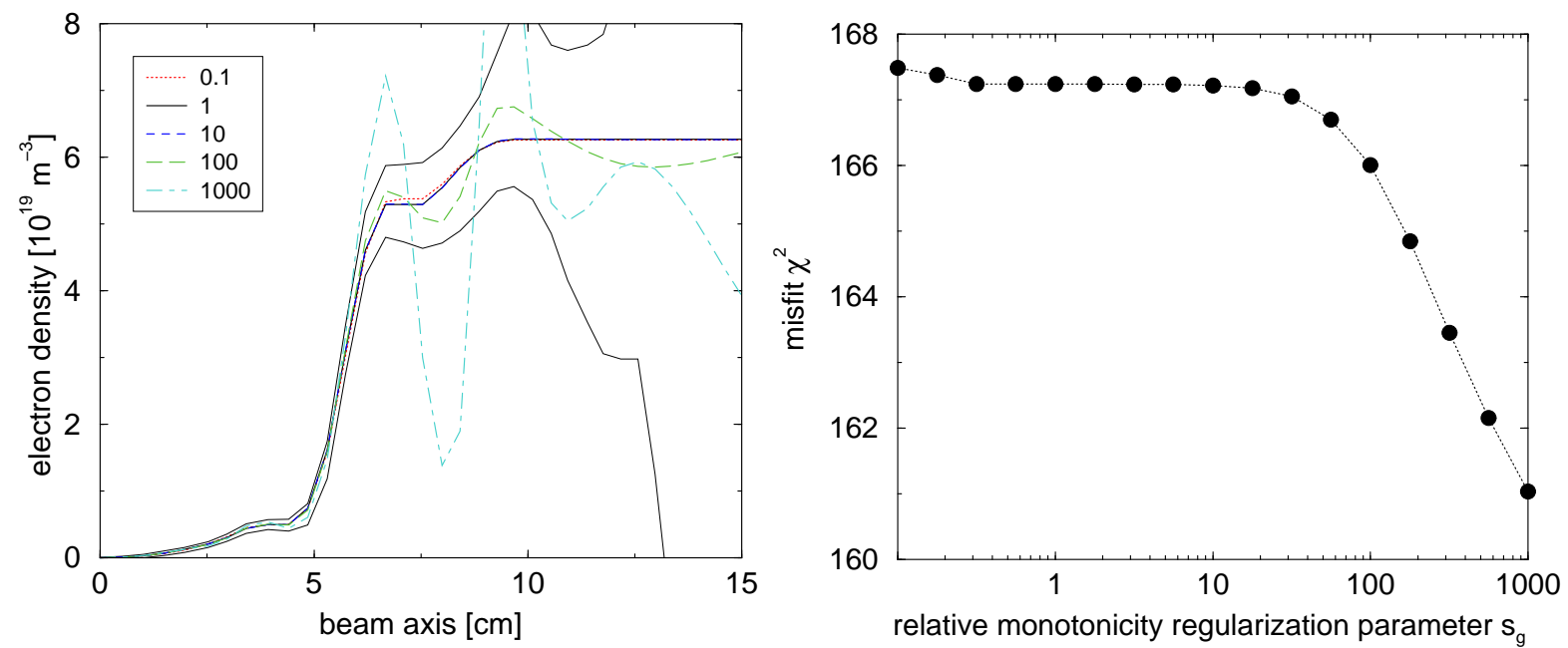

Figure 16. Left: Density profiles obtained with 5 different values for the regularization parameter $s_{g}$ penalizing deviations from monotonicity. The monotonicity constraint becomes weaker for increasing values of $s_{g}$. Right: $\chi^{2}$-misfit as a function of the regularization parameter.

should be approximately about the number of data $(5 * 35=175)$ minus the number of effective parameters $(<14)$. The profiles for $s_{g}=100,1000$ are subject to strong oscillations which are considered to have no physical cause. A sensitivity analysis of the dependence of the profiles on $s_{g}$ and the respective residues allows to study if the nonmonotonic profile structures might be due to real physical effects, e.g. hollow profiles, or if there are systematic uncertainties in the data or in the modeling which have to be resolved.
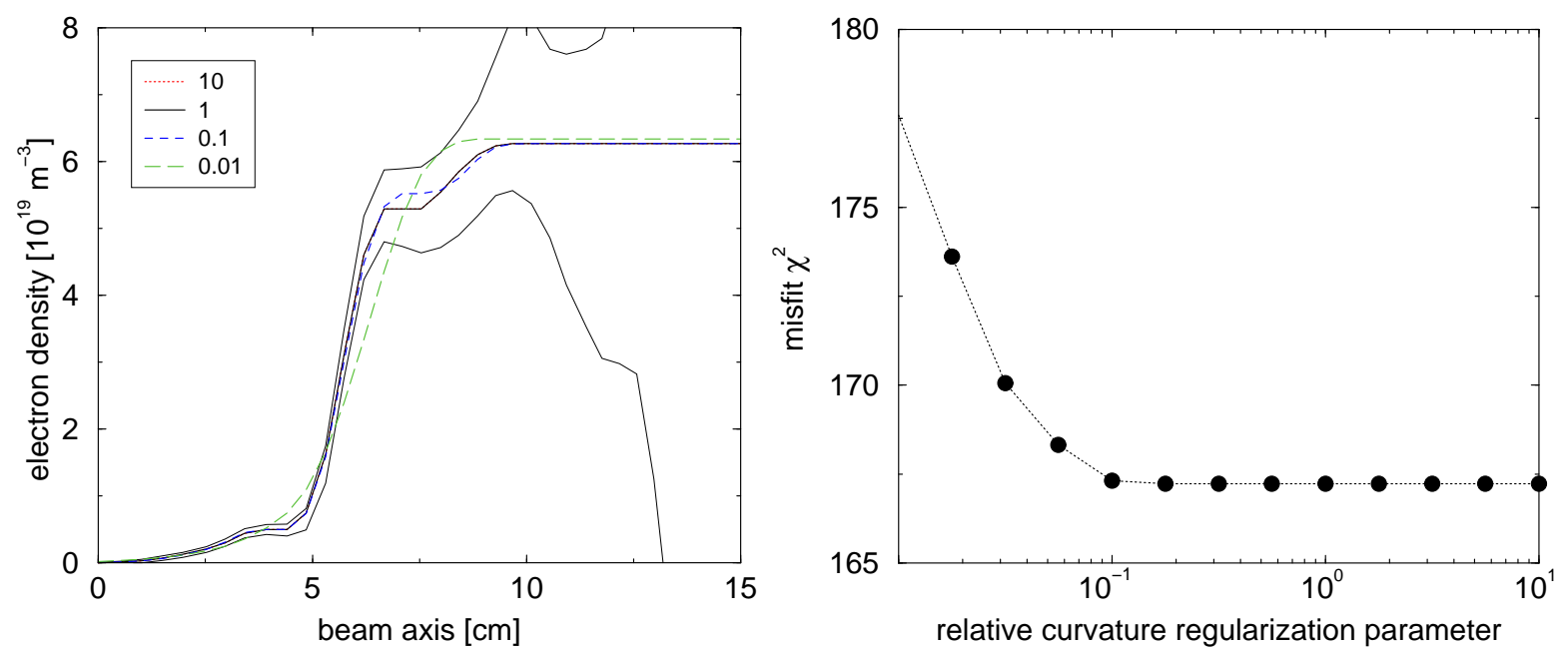

Figure 17. Left: Density profiles obtained with 4 different values for the regularization parameter penalizing profile curvature. The curvature penalization increases with decreasing values of the regularization parameter. Right: $\chi^{2}$-misfit as a function of the regularization parameter. 
The second prior used penalizes density profile curvature which is a natural approach for cubic splines [29]. The left panel of figure 17 shows density profiles obtained with 4 different values for the curvature regularization parameter $s_{c}$. The routinely used value is $s_{c}=1(\times 0.5)$ for which also the profile error bars are shown. The profiles for $s_{c}=0.1,1,10$ have a low degree of curvature penalization and are hardly to be separated. The right panel depicts the corresponding $\chi^{2}$-misfit values. For $s_{c}>0.1$ the decrease of the $\chi^{2}$-misfit with the increase of $s_{c}$ is small showing that the choice of $s_{c}=1$ is robust. For $s_{c}<0.1$ the misfit increases rapidly with decreasing $s_{c}$ because the data are over-smoothed and significant structures in the data are lost. Hence, the value $s_{c}=1$ selected for routine analysis appears to provide robust profile smoothness with margins for fitting data structures.

\section{Profile uncertainties}

Profile uncertainties are a necessary requisite to evaluate the quality of the estimated profile. Frequently, an error band or confidence interval is given. However, it is often not clear how to interpret this interval. For example, how should the confidence interval being used if one is interested in derived profiles, e.g. $p_{e}=n_{e} T_{e}$, in fitting of physicallymotivated analytical functions together with other diagnostics results, or in using the profiles in plasma modeling, e.g. equilibrium codes? In general, the problem with such one-dimensional uncertainty measures for all kind of distributions is that they can be produced in different ways. If a local measurement, e.g. Thomson scattering, is analyzed, then the error bar directly reflects the estimation uncertainty of the parameters for the corresponding observed plasma volume. For these local measurements, and the corresponding local analysis, correlations between parameters of different channels are not present which simplifies the interpretation of uncertainties. The situation changes, if, for a local measurement, an analytical function is fitted to all measured channels simultaneously, or if the interpretation of the measurement is non-local. The latter occurs for the analysis of the lithium beam where the local emission intensity depends on the plasma the beam has passed. The estimated densities at the different spatial channels are correlated resulting in correlated estimation uncertainties. Additional to the correlation in data interpretation given by the likelihood pdf, constraints or prior information can further imply correlations in the parameters, e.g. (weak) monotonicity constraints. The result of employing such additional information is a non-trivial correlation structure in the profile properties. Such correlations can not be quantified and visualized with confidence intervals. In particular, for further processing of the profiles more elaborate techniques have to be used to consider the full correlation structure.

Figure 18 compares profile uncertainties obtained with four different methods. Panel 1 (leftmost) shows a confidence interval obtained with the Laplace approximation. The Laplace approximation is a second-order Taylor expansion of the logarithm of the posterior pdf at the posterior maximum. The estimated parameter uncertainty is a 

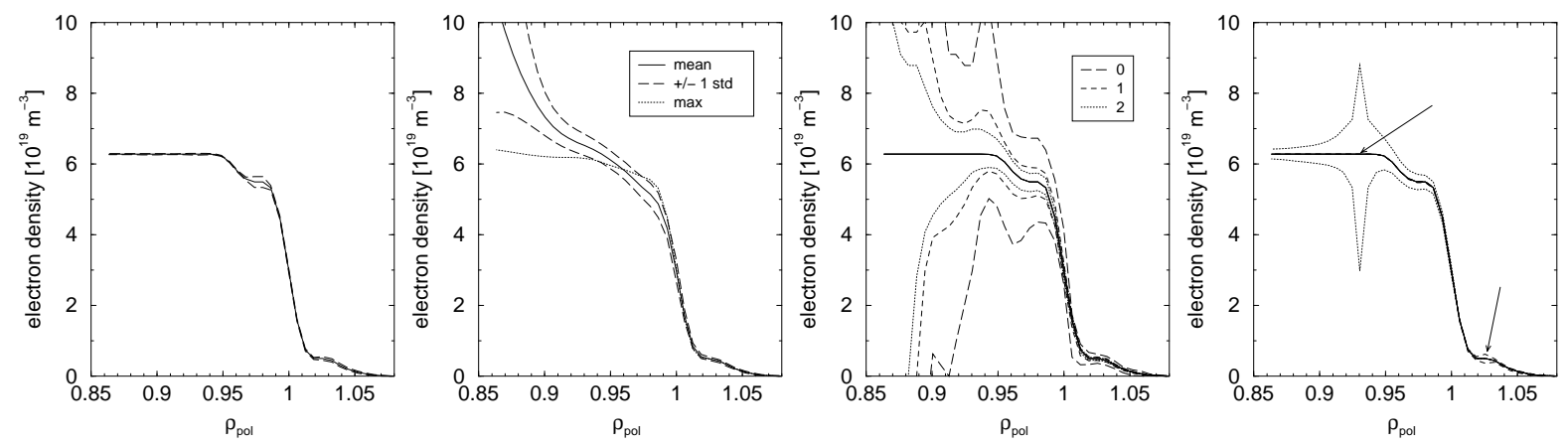

Figure 18. Comparison of density profile uncertainties obtained with the Laplace approximation (error propagation employing the Hessian matrix) (left panel), $\chi^{2}$ method for different correlation lengths (middle), and error stars at two different spatial positions (right).

property of the curvature of the posterior pdf at the maximum and, hence, does not account for the global behavior. In the present case, the error band estimated with the Laplace approximation is small. The error band is mainly determined by the prior pdf penalizing non-monotonic density profiles. Therefore, in case of strong asymmetric probability distributions it is not useful to employ a symmetric measure for uncertainties which does not describe the overall behavior of the posterior pdf.

The overall behavior of the posterior pdf can be revealed by Markov-Chain Monte Carlo (MCMC) methods. Panel 2 shows results from a MCMC run where the mean of the density samples and the corresponding upper and lower \pm 1 standard deviation is shown. Please note the asymmetric confidence band. The dotted curve shows one of the 500000 MCMC samples (50 min computation time on a single CPU), namely the most probable density profile. The large error band compared to the Laplace approximation and the difference between the most probable and the mean profile depicts the asymmetry of the posterior pdf with respect to the properties at the posterior mode (maximum). By calculating histograms of properties of the MCMC profile samples one can easily produce marginal posterior pdfs of any quantity of interest.

The resulting profiles often depend sensitively on the prior pdfs provided. In our case, the monotonicity prior has the most prominent effect. To depict the variability of the profiles given the information content of the data only, panel 3 shows the uncertainties derived from a method which increases/decreases single or neighboring groups of density values until an increment of $\chi^{2}$ by one is obtained [32]. The longdashed error band shows the interval for which single density value out of the 35 spatial channels are increased (upper band) or decreased (lower band) such that the misfit of the data $\left(\chi^{2}\right)$ becomes worse by $\Delta \chi^{2}=1$. This measure is useful since an increase of one corresponds to \pm 1 standard deviation uncertainty of the corresponding data value. This measure works only for the present case of calibrated data errors (Poisson statistics). The error band obtained in this way is quite large. The dashed and dotted curves show error bands for correlated neighboring density channels. For the dashed 
(dotted) band 3 (5) neighboring density values are jointly scaled to obtain $\Delta \chi^{2}=1$. With increasing correlation length the error band decreases which can be depicted in an error star [32]. Panel 4 (rightmost) shows an error stars at two different spatial positions which shows the estimation uncertainty for different correlation lengths. At $\rho_{\text {pol }}=0.93$ the density estimate has an uncertainty of about $50 \%$ if one changes a single channel only, but the uncertainty shrinks to about $3 \%$ if one looks for the uncertainty of the plateau value. The error stars are suitable for depicting correlation properties in the uncertainties. If the method of error stars is applied to the likelihood $\left(\chi^{2}\right)$ only, it neglects physical constraints, e.g. monotonicity, completely. However, it can be applied to the full posterior pdf, where the non-trivial correlation is considered.

In general, summarizing the complex uncertainty structure in single quantities has to be treated carefully. In the probabilistic approach, the only exhaustive result of the analysis is given by the posterior pdf. The posterior pdf contains the complete knowledge about the parameters of interest including all types of correlations. If the profile has to be used in subsequent processing without loss of information, one has to use the full posterior pdf as prior pdf for the following analysis. The uncertainties of the profiles propagate correctly if, and only if, the full posterior pdf is used. Alternatively, one can try to derive the quantity of interest directly from all measured data (Integrated Data Analysis) by combining the likelihoods of all data, all prior pdfs from additional information and the modeling where the physical quantities of interest enter.

\section{Summary}

A new probabilistic data analysis tool for analyzing Li-beam emission profiles was developed. The probabilistic description of the data benefits from a thorough error analysis of all data involved. Prior information about weak constraints on monotonicity are useful. No direct inversion occurs. In comparison to the old algorithm it allows to analyze density profiles for any density regime with a temporal resolution of $50 \mu \mathrm{s}$. The benefit of the achieved temporal resolution is shown with ELM resolved measurements. For small densities the profiles are limited by the actual spatial distribution of the measurement channels in the plasma and not due to beam attenuation. The improved method allows to measure edge pedestal densities up to $7 \times 10^{19} \mathrm{~m}^{-3}$. For medium and large densities the reliable density region as well as the upper density limit can be extended by larger beam energies. The dependence of the density profiles on electron temperature or impurity content is negligible. Profile uncertainty measures can not be provided for general purpose because they reflect only reduced properties of the posterior pdf. One always should prefer to work with the full posterior pdf in an integrated framework.

\section{References}

[1] K. Kadota, K. Tsuchida, Y. Kawasumi, and J. Fujita. Plasma Phys., 20:1011, 1978. 
[2] K. McCormick, M. Murmann, and M. El Shaer. J. Nucl. Mater., 121:48, 1984.

[3] K. McCormick and the ASDEX team. Rev. Sci. Instuments, 56:1063, 1985.

[4] J. Schweinzer, E. Wolfrum, F. Aumayr, M. Pöckl, HP. Winter, R. P. Schorn, E. Hintz, and A. Unterreiter. Reconstruction of plasma edge density profiles from LiI $(2 \mathrm{p})$ emission profiles. Plasma Phys. Control. Fusion, 34:1173-1183, 1992.

[5] K. McCormick, S. Fiedler, G. Kocsis, J. Schweinzer, and S. Zoletnik. Edge density measurements with a fast Li beam probe in tokamak and stellarator experiments. Fusion Engineering and Design, 34-35:125-134, 1997.

[6] S. Fiedler, R. Brandenburg, J. Baldun, K. McCormick, F. Aumayr, J. Schweinzer, HP. Winter, and W7-AS and ASDEX Upgrade Team. Edge plasma diagnostics on W7-AS and ASDEX Upgrade using fast Li beams. J. Nucl. Mater., 266-269:1279-1284, 1999.

[7] M. Brix, A. Korotkov, M. Lehnen, P. Morgan, K. McCormick, J. Schweinzer, D. Summers, J. Vince, and JET EFDA Contributors. Determination of edge density profiles in JET using a $50 \mathrm{kV}$ lithium beam. In 28th EPS Conference on Controlled Fusion and Plasma Physics. Contributed Paper, pages 389-392, 2002.

[8] K. Nakamura, H. Iguchi, J. Schweinzer, A. Shimizu, M. Isobe, C. Takahashi, S. Nishimura, C. Suzuki, Y. Yoshimura, K. Nagaoka, T. Minami, T. Akiyama, K. Ida, K. Matsuoka, and S. Okamura. Two-dimensional plasma structure in the edge region of the compact helical system. Nucl. Fusion, 47:251-256, 2007.

[9] R. Fischer, A. Dinklage, and E. Pasch. Bayesian modelling of fusion diagnostics. Plasma Phys. Control. Fusion, 45:1095-1111, 2003.

[10] Z. A. Pietrzyk, P. Breger, and D. D. R. Summers. Deconvolution of electron density from lithium beam emission profiles in high edge density plasmas. Plasma Phys. Control. Fusion, 35:1725$1744,1993$.

[11] J. Schweinzer, D. Wutte, and HP. Winter. A study of electron capture and excitation processes in collisions of multiply charged ions with lithium atoms. J. Phys. B: At. Mol. Opt. Phys., 27:137-153, 1994.

[12] R. Brandenburg, J. Schweinzer, F. Aumayr, and HP. Winter. Li(2p-2s) excitation by slow ions. J. Phys. B: At. Mol. Opt. Phys., 31:2585-2599, 1998.

[13] R. Brandenburg, J. Schweinzer, S. Fiedler, F. Aumayr, and HP. Winter. Modelling of fast Li beams for fusion edge plasma diagnostics. Plasma Phys. Control. Fusion, 41:471-484, 1999.

[14] J. Schweinzer, R. Brandenburg, I. Bray, R. Hoekstra, F. Aumayr, R.K. Janev, and HP. Winter. Database for inelastic collisions of Lithium atoms with electrons, protons and multiply charged ions. Atomic Data \& Nuclear Data Tables, 72:239-273, 1999.

[15] K. McCormick, S. Fiedler, G. Kocsis, J. Schweinzer, and S. Zoletnik. Edge density measurements with a fast Li beam probe on tokamak and stellarator experiments. Technical Report IPPIII/211, Max-Planck-Institut für Plasmaphysik, Garching, 1996.

[16] I. Nunes, M. Manso, F. Serra, L.D. Horton, G.D. Conway, A. Loarte, the ASDEX Upgrade, and CFN Reflectometry Teams. Density profile analysis during an ELM event in ASDEX Upgrade H-modes. Nucl. Fusion, 45:1550-1556, 2005.

[17] R. Fischer, C. Wendland, A. Dinklage, S. Gori, V. Dose, and the W7-AS team. Thomson scattering analysis with the bayesian probability theory. Plasma Phys. Control. Fusion, 44:1501-1519, 2002 .

[18] R. Fischer and V. Dose. Electron energy distribution reconstruction in low-pressure helium plasmas from optical measurements. Plasma Phys. Contr. Fusion, 41:1109, 1999.

[19] M. Krychowiak, R. König, T. Klinger, and R. Fischer. Bayesian analysis of the effective charge from spectroscopic bremsstrahlung measurement in fusion plasmas. J. Appl. Phys., 96:47844792, 2004 .

[20] H. Meister, R. Fischer, L.D. Horton, C.F. Maggi, D. Nishijima, ASDEX Upgrade Team, C. Giroud, K.-D. Zastrow, and JET-EFDA Contributors. Zeff from spectroscopic bremsstrahlung measurements at ASDEX Upgrade and JET. Rev. Sci. Instrum., 75:4097-4099, 2004. 
[21] V. Dose, J. Neuhauser, B. Kurzan, H. Murmann, H. Salzmann, and ASDEX Upgrade Team. Tokamak edge profile analysis employing Bayesian statistics. Nuclear Fusion, 41:1671-1685, 2001.

[22] R. Fischer. Bayesian group analysis of plasma-enhanced chemical vapour deposition data. New J. Phys., 6:25, 2004.

[23] R. Preuss and A. Dinklage. Model comparison in plasma energy confinement scaling revisited. In A. Mohammad-Djafari, editor, Bayesian Inference and Maximum Entropy Methods in Science and Engineering, volume Conf. Proc. 872, pages 280-287. AIP, Melville, NY, 2006.

[24] A. Dinklage, R. Fischer, and J. Svensson. Topics and methods for data validation by means of Bayesian probability theory. Fusion Science and Technology, 46:355-364, 2004.

[25] H. Dreier, A. Dinklage, R. Fischer, M. Hirsch, P. Kornejew, and E. Pasch. Bayesian design of diagnostics: Case studies for Wendelstein 7-X. Fusion Science and Technology, 50:262-267, 2006.

[26] A. Dinklage, R. Fischer, H. Dreier, J. Svensson, and Yu. Turkin. Integrated approaches in fusion data analysis. In R. Fischer, R. Preuss, and U. von Toussaint, editors, Bayesian Inference and Maximum Entropy Methods in Science and Engineering, volume Conf. Proc. 735, pages 43-51, Melville, NY, 2004. AIP.

[27] D. S. Sivia and J. Skilling. Data Analysis - A Bayesian Tutorial. Oxford University Press, Oxford, 2006.

[28] D. Wutte, R.K. Janev, F. Aumayr, M. Schneider, J. Schweinzer, J. Smith, and HP. Winter. Cross sections for collision processes of $\mathrm{Li}$ atoms interacting with electrons, protons, multiply charged ions, and hydrogen molecules. Atomic Data \&3 Nuclear Data Tables, 65:155-180, article No. DT970736, 1997.

[29] R. Fischer, K. M. Hanson, V. Dose, and W. von der Linden. Background estimation in experimental spectra. Phys. Rev. E, 61:1152, 2000.

[30] L. Giannone, A.C.C. Sips, O. Kardaun, F. Spreitler, W. Suttrop, and the ASDEX Upgrade Team. Regime identification in ASDEX Upgrade. Plasma Phys. Control. Fusion, 46:835-856, 2004.

[31] R. Fischer, W. von der Linden, and V. Dose. On the importance of $\alpha$ marginalization in maximum entropy. In R. N. Silver and K. M. Hanson, editors, Maximum Entropy and Bayesian Methods, page 229. Kluwer Academic Publishers, Dordrecht, 1996.

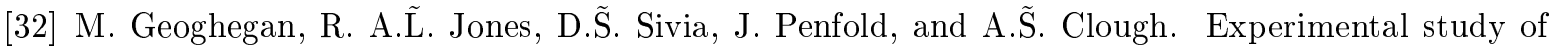
surface segregation and wetting in films of a partially miscible polymer blend. Phys. Rev. E, $53: 825-837,1996$. 